\title{
Parallel Motion Processing for the Initiation of Short-Latency Ocular Following in Humans
}

\author{
Guillaume S. Masson and Eric Castet \\ Centre de Recherche en Neurosciences Cognitives, Centre National de la Recherche Scientifique, FRE2098, \\ 13402 Marseille, France
}

\begin{abstract}
With the scleral search coil technique, we recorded ocular following responses elicited by either grating or plaid pattern motions. Grating motion elicited tracking responses at short latencies ( $~ 85 \mathrm{msec})$. Type I plaid motion made by summing two orthogonal moving gratings elicited ocular following with identical short latencies. Trial-by-trial vector decomposition showed that plaid-driven responses were best predicted by a vector average of the component-driven responses. Similar results were found with micropatterns made of 16 Gabor patches with drifting carriers of two different orientations. "Unikinetic" plaids were constructed by summing a moving and stationary grating, with a $45^{\circ}$ orientation difference, so that component and pattern motion directions were separated by $45^{\circ}$. Eye movements exhibited two components. Ocular follow-
\end{abstract}

In primates, short-latency ocular following responses help vision by stabilizing the image of the object of interest onto the retina (for review, see Miles, 1998). The response properties reflect many of the signatures of early motion processing (Miles et al., 1986; Gellman et al., 1990; Masson et al., 2000, 2001) and, in monkeys, they depend on neural activity in cortical visual areas MT and MST (Kawano, 1999). This sensorimotor transformation relies on a motion integration mechanism that reconstructs the two-dimensional (2D) velocity of the object motion from the different motion cues present in the image (Masson et al., 2000) and therefore offers an exquisite tool to dissect the mechanisms of early visual motion processing in the primate brain.

There is behavioral (Masson et al., 2000), psychophysical (Lorenceau et al., 1993), and physiological (Pack and Born, 2001) evidence that $2 \mathrm{D}$ motion computation is a dynamical process that takes several tens of milliseconds to be accurately completed. In a previous study, we showed that, in humans, short-latency ocular following of a moving grating presented behind an elongated and oblique aperture (the so-called "barber pole phenomenon") has two components : an early (latency $\sim 85 \mathrm{msec}$ ) component driven only by grating motion, and a later (latency $\sim 110 \mathrm{msec}$ ) compo-

\footnotetext{
Received Jan. 22, 2002; revised March 20, 2002; accepted March 21, 2002.

This work was supported by grants from the Centre National de la Recherche Scientifique, the French Ministère de la Recherche (ACI-2000-5052), and the Fondation pour la Recherche Médicale to G.S.M. We thank B. Arnaud, R. Fayolle, and A. DeMoya for technical assistance, and D. R. Mestre for performing the experiments. We thank Lee Stone, Brent Beutter, and Bill MacKay for a critical reading of a previous version of this manuscript and the reviewers for their very helpful comments.

Correspondence should be addressed to Dr. Guillaume S. Masson, Centre de Recherche en Neurosciences Cognitives, Centre National de la Recherche Scientifique FRE2098, 31 Chemin Joseph Aiguier, 13402 Marseille, cedex 20, France. E-mail: masson@lnf.cnrs-mrs.fr.

Copyright (ㄷ) 2002 Society for Neuroscience $\quad 0270-6474 / 02 / 225149-15 \$ 15.00 / 0$
}

ing was first initiated in the grating motion direction, at ultrashort latency. A second component was initiated $\sim 20 \mathrm{msec}$ later, curving the responses toward the pattern motion direction. The later component was specifically, and independently, affected by both relative spatial frequency and contrast between component gratings. The early response components showed a much steeper contrast response function than the late component. These results suggest that initial ocular following is underpinned by parallel processing of component- and pattern-related velocities followed by an integrative stage that computes the two-dimensional surface motion.

Key words: ocular tracking; plaid motion; Fourier; nonFourier; parallel processing; $2 D$ visual motion integration nent driven by $2 \mathrm{D}$ features such as line-endings generated at the intersections between the grating and the aperture edges (Masson et al., 2000). Interestingly, Pack and Born (2001) subsequently showed that direction selectivity of macaque MT neurons exhibits a similar temporal evolution when presented with a set of oblique moving lines : neurons first sense the motion orthogonal to the bars, but $\sim 60 \mathrm{msec}$ later encode the true $2 \mathrm{D}$ global motion of the bars. In monkeys, smooth pursuit eye movements exhibit a similar temporal evolution, although on a slower time scale (Pack and Born, 2001).

These results have two major outcomes : (1) an understanding of $2 \mathrm{D}$ motion processing requires consideration of its temporal evolution, and (2) tracking eye movements offer a unique opportunity to dissect the dynamics of the underlying neural solution. To probe the dynamics of $2 \mathrm{D}$ motion processing, we recorded in humans the short-latency, initial (open-loop) part of the ocular following responses elicited by different types of drifting plaids. Plaids are 2D patterns constructed by summing two sinusoidal gratings of different orientations (Adelson and Movshon, 1982). They have been extensively used to probe $2 \mathrm{D}$ motion computation (Wilson et al., 1992) and its neural implementation (Movshon et al., 1985; Rodman and Albright, 1989). In the present study, we used plaid motion to tease apart the contribution and the timing of both grating and pattern motion processing. We demonstrate that these two types of motion signals are processed independently, with different latencies, but are combined together so that at the end of the open-loop period of ocular following responses, the $2 \mathrm{D}$ tracking direction can be predicted by a vector combination calculation.

\section{MATERIALS AND METHODS}

Subjects. Three subjects [two naive and one author (G.M.)] participated in the complete study. A smaller set of additional data were collected on 
two other naive subjects to ensure that similar results were obtained in the most critical experiments. Because data from these two subjects were not different, they are not reported herein. All subjects were free of neurological or ophthalmological diseases and had eye examinations before participating in the experiments. All subjects had normal or corrected-to-normal acuity.

Eye movement recording and visual stimuli. Most techniques have been described previously (Masson et al., 2000). Eye movements were recorded using the electromagnetic search-coil technique (Robinson, 1963) with coils embedded in a Silastin scleral ring (Collewijn et al., 1985). Coils were placed in one eye after application of 1-2 drops of anesthetic (Novesine; Merck, Paris, France). Daily wearing time was limited to $\sim 60$ min. Data acquisition, on-line control of the behavior, and stimulus triggering were controlled by a personal computer (PC) using the REX software package under the real-time QNX operating system (Hays et al., 1982). Voltage signals separately encoding horizontal and vertical positions of the right eye were low-pass filtered (Bessel, 6 poles, DC $-180 \mathrm{~Hz}$ ) and sampled at $1 \mathrm{kHz}$, with a resolution of 16 bits.

Subjects were seated in a fiberglass chair, with chin and head rests, and faced a vertical screen (viewing distance: $1 \mathrm{~m}$; subtense: $70 \times 70^{\circ}$ ) onto which visual stimuli were back-projected from a high-resolution Electrohome Marquee 1800 trichromatic video projector (refresh rate: $76 \mathrm{~Hz}$ ). The luminance output of the back-projection system was calibrated to correct for its gamma nonlinearity using a lookup table. Visual stimuli consisted of 24 frame movies, precomputed using the HIPS libraries (Landy et al., 1984) and stored in the memory of an Octane SGI workstation. The visual workstation and experimental PC communicated through a serial port. Synchronization between the two computers has been fully described earlier (Masson et al., 2000) and resulted in a 16 msec jitter around the selected post-saccadic delay and a $3 \mathrm{msec}$ jitter around the time 0 of stimulus onset. The first jitter affects the actual post-saccadic delay between the end of the saccade and the onset of stimulus motion. The main consequence of this is a larger variability in the amplitude of the responses, a parameter known to be a function of post-saccadic delay (Kawano and Miles, 1986; Gellman et al., 1990). The second jitter introduces a small $( \pm 3 \mathrm{msec})$ variability in the measurement of the response latency, relative to the actual timing of stimulus motion onset.

All grating and plaid motion stimuli were presented within a circular aperture (diameter: $20^{\circ}$ ). The stimulus surround was a gray-level background with the same mean luminance as the motion stimuli, which covered $70 \times 56^{\circ}$ of visual angle. Spatiotemporal properties of the moving gratings were kept constant [spatial frequency: 0.27 cycles per degree (cpd); temporal frequency: $6 \mathrm{~Hz}$; drifting speed: $23 \%$ sec] across conditions, except in the last experiments in which contrast or spatial frequencies were the parameters of interest. Mean grating luminance was $22.25 \mathrm{~cd} / \mathrm{m}^{2}$, and Michelson's contrast was $80 \%$. Grating motion stimuli were always interleaved with plaid motion stimuli. Two different types of moving plaids were generated. First, two perpendicular, moving gratings were summed together to form a type I plaid whose pattern direction fell between the component gratings' directions. Second, unikinetic plaids were constructed by the sum of one stationary and one moving grating whose orientations differed by $45^{\circ}$ (Goréa and Lorenceau, 1991; Dobkins et al., 1998). Thus, grating and pattern motion directions differed by $45^{\circ}$. These unikinetic plaids are a limiting case of the type II plaids, which were originally defined in terms of velocity direction vectors (Ferrera and Wilson, 1990) With unikinetic plaids, grating motion is the only source of Fourier motion, but a non-Fourier motion component is also generated whose direction depends on relative orientation and spatial frequency (Wilson and Kim, 1994). Therefore, by keeping grating motion direction along one of the four cardinal axes, the contribution of any non-Fourier motion can be clearly identified by looking at ocular responses along the orthogonal axis. Hence, unikinetic plaids offer the purest opportunity to probe the relative contribution of Fourier and non-Fourier motion to tracking initiation. For all but the last experiment, total contrast and mean luminance of plaid and grating stimuli were equal $(80 \%, 22.25$ $\left.\mathrm{cd} / \mathrm{m}^{2}\right)$. In the last experiment, the contrasts of both gratings were manipulated. Contrast of the static grating was varied from 2.5 to $80 \%$, while contrast of the moving grating was varied complementarily from 97.5 to $20 \%$, so that total contrast of the plaid pattern remained constant at $100 \%$. Notice that over the range $20-97.5 \%$, the contrast of the moving grating was well above saturation values found for ocular following responses when the contrast of moving gratings presented alone was varied.

To test the linear combination of motion signals that are nonoverlap- ping in space, we ran one experiment with Gabor micropattern motion stimuli (Boulton and Baker, 1991). Motion stimuli were generated by a $4 \times 4$ array of small, symmetrical (diameter: $2.75^{\circ}$ ) Gabor patches, each one being formed by the multiplication of a moving sinusoidal carrier and a stationary two-dimensional Gaussian window. Carrier spatial and temporal frequencies were of $0.72 \mathrm{cpd}$ and $12 \mathrm{~Hz}$, respectively (i.e., carrier speed: $\left.18^{\circ} / \mathrm{sec}\right)$. With unikinetic micropatterns, all motion signals were in the same direction. With bikinetic micropatterns, the array was divided into two groups of Gabor patches, with carriers moving in orthogonal directions. Micropatterns formed a square array with 16 local motion signals having either identical or orthogonal directions, covering $11 \times 11^{\circ}$ of the visual field.

Behavioral paradigm. The behavioral paradigm has been extensively described previously (Miles et al., 1986; Gellman et al., 1990). Trials started with a gray background of mean luminance $\left(22.5 \mathrm{~cd} / \mathrm{m}^{2}\right)$ and a target spot produced by a light-emitting diode (LED) was back-projected onto the screen, $10^{\circ}$ to the right of center. The subject was required to fixate this spot for a randomized time interval, after which the spot disappeared and a second spot appeared at the center of the screen. The subject was required to make a saccadic eye movement to this new target, at which time it was switched off. With gaze now directed at the center of the screen, after a post-saccadic delay of $50 \mathrm{msec}$, the motion stimulus was presented for $220 \mathrm{msec}$ before the screen was blanked, ending the trial. In the different experiments, all conditions were fully randomized and interleaved with catch-trials where no motion stimulus was presented. Using an initial gray-level background was a slight modification of the original paradigm used by Miles et al. (1986), in which identical random-dot patterns are presented before (i.e., static) and after (i.e., moving) the centering saccade (Gellman et al., 1990). This is because we wanted to avoid any static, pre-saccadic cueing about the next motion stimulus (e.g., grating versus plaid). By doing so, we avoided anticipatory drifting eye movements as well as anticipatory shift of attention toward a specific part of the stimulus (e.g., blobs at the intersection between the two gratings).

Data analysis. In a given experiment, it was usual to collect data until each condition had been repeated more than $\sim 100$ times, permitting good resolution of the responses to be achieved through averaging. After several daily recording sessions, all data were transferred to an SGI workstation for off-line analysis. Eye position data were linearized with a fifth-order polynomial function derived from a calibration procedure before each session, and were then smoothed with a cubic spline of weight $10^{6}$ (Busettini et al., 1991). All subsequent analyses used these splined data. Rightward and upward eye movements were defined as positive. Eye velocity signals were computed with a two-point differentiation. For a given experimental condition, all single trials were simultaneously displayed with interactive visual software to remove remaining small saccadic eye movements, to extract average horizontal and vertical eye velocity profiles, and to compute amplitude measurements and latencies in both horizontal and vertical domains on a trial-by-trial basis. Ocular following was triggered in the close temporal vicinity of a saccade. Therefore, to eliminate any effects caused by post-saccadic drift, all data shown have the saccade-only condition subtracted.

Quantitative analysis was done by measuring for each trial the changes in vertical $\left(\Delta \mathrm{e}_{\mathrm{v}}\right)$ and horizontal $\left(\Delta \mathrm{e}_{\mathrm{h}}\right)$ position over two $40 \mathrm{msec}$ time windows starting either at 95 or at $135 \mathrm{msec}$ after stimulus onset. These two time windows did not overlap and were selected to quantify the amplitude changes in both early and late phase of the responses, while remaining in the open-loop period (twice the latency). Mean ( $\pm \mathrm{SE}$ ) was then computed for each condition, and we present data for each subject. In Figures 12 and 14, to remove idiosyncratic differences in amplitude, changes in position were normalized with the following formula :

$$
R_{\mathrm{n}}=\left[R_{\mathrm{i}}-R_{\min }\right] /\left[R_{\max }-R_{\min }\right]
$$

where $R_{\max }$ and $R_{\min }$ are the maximum and the minimum response amplitude across the conditions, and $R_{\mathrm{i}}$ is a given data point.

Because we recorded 2D tracking responses to either 1D (grating) or 2D (plaid) motion stimuli, the best way to describe the oculomotor performance is by computing a vector describing the responses. For each trial, tracking directions were computed for each time window as being equal to $\tan ^{-1}\left(\Delta \mathrm{e}_{\mathrm{v}} / \Delta \mathrm{e}_{\mathrm{h}}\right)$. Distribution histograms of response directions were computed for each subject and stimulus condition. To test whether ocular following to plaid motion can be predicted from the responses to grating motion only, we applied the method used by Lisberger and Ferrera (1997) and by Ferrera (2000) to predict smooth pursuit responses 
to two target stimuli from responses to one target stimuli. For each trial recorded with a type I plaid, the $2 \mathrm{D}$ response vector $(R p)$ during the 95-135 msec time interval was decomposed as the weighted sum of the average 2D response vectors $\left(R g_{1}, R g_{2}\right)$ gathered with each grating component, independently:

$$
R p=\omega_{1} \cdot R g_{1}+\omega_{2} \cdot R g_{2}
$$

Distributions of each vector weight $(\omega)$ were computed across all trials for plaid motion directions, and the mean of the distribution was estimated from the best fitting Gaussian function. When $\omega_{1}=\omega_{2} \approx 1$, responses to plaid can then be predicted from a vector sum of the responses to the components; when $\omega_{1}=\omega_{2} \approx 0.5$, best prediction is done by a vector average computation. When one of the weights is distributed around 0 while the other is distributed around 1 , the response to a plaid motion is dominated by one of the components. In this case, the distribution can be either unimodal or bimodal, indicating that ocular following responses are always driven by the same component or by an alternation between one or the other component, respectively (Ferrera, 2000). Similar computation was done to predict the ocular following to bikinetic micropatterns from the mean responses to component monokinetic micropatterns.

Unikinetic plaids were generated by adding one moving grating and one stationary grating. Three directions can be predicted with each type of unikinetic plaid motion : the first-order (Fourier) motion direction is the direction of the moving grating. The Intersection of Constraints (IOC) rule (Adelson and Movshon, 1982) is a geometric construction that indicates the true pattern motion direction from the velocity vectors orthogonal to the grating orientations. For unikinetic plaids, the IOC direction is given by the orientation of the stationary grating, i.e., $45^{\circ}$ away from the grating motion direction (Goréa and Lorenceau, 1991). Finally, there are second-order (non-Fourier) motion signals that indicates the direction of motion of texture boundaries (Wilson et al., 1992; Wilson, 1999). Their direction can be computed (Wilson et al., 1992; Wilson and Kim, 1994) if one assumes that second-order motion is extracted through a filter-rectify-filter mechanism (Chubb and Sperling, 1988). With two grating components of the same spatial frequency $\omega$ and orientation difference $\Theta=45^{\circ}$, the squaring produces four new spatial frequencies, from the product of the two gratings and the squares of the component gratings. The latter have the same orientation as the component gratings, but twice their spatial frequency $(\omega)$. The product of the component gratings produces two new gratings of spatial frequencies $\omega_{\mathrm{p} 1}=2 \cdot \omega \cdot \cos (\Theta)$ and $\omega_{\mathrm{p} 2}=2 \cdot \omega \cdot \sin (\Theta)$ at right angles (Wilson et al., 1992). The vector sum of these non-Fourier motions is in the same direction as the pattern motion (i.e., $45^{\circ}$ away from the grating motion). Moreover, given our set of experimental conditions, the direction of the vector sum between first- and second-order motion signals was $\sim 33^{\circ}$ away from the grating motion direction.

Horizontal and vertical latencies were estimated for each trial, through a criterion-free method described earlier for smooth pursuit eye movements (Carl and Gellman, 1987) and adapted for ocular following responses (Gellman et al., 1990; Masson et al., 2000). Briefly, the horizontal (vertical) latency was defined as the intersection of two regression lines fitted through a baseline $40 \mathrm{msec}$ time window (starting $20 \mathrm{msec}$ after stimulus onset) and a $40 \mathrm{msec}$ response window (starting when eye velocity exceeded 4 SD of the mean measured from the baseline interval) of horizontal (vertical) eye velocity. When the latency in either horizontal or vertical domains was not measurable, the trial was rejected. Therefore, although vertical and horizontal response latencies were measured independently, analysis was conducted on both mean vertical and horizontal latencies, and the latency difference, on a trial-by-trial basis.

\section{RESULTS}

We first describe the ocular following responses to type I plaid stimuli where pattern motion direction can be extracted from a linear combination of the motion directions of the two component gratings. To further probe the linear combination of motion signals, we then compare the ocular tracking of micropatterns made of either a single or two different Fourier motions. Then, to separate the relative contribution of grating and pattern motion signals, we report responses to a particular instance of type II plaids (Ferrera and Wilson, 1990), the unikinetic plaid patterns (Goréa and Lorenceau, 1991; Dobkins et al., 1998) where these two motions are in different directions. Throughout the study, we used specific grating orientations that enabled us to project the relative contribution of grating and pattern motions onto the orthogonal axes of horizontal and vertical eye movements. By doing so, we were able to measure latencies and latency differences between component- and pattern-driven eye movements (Masson et al., 2000).

\section{Responses to single grating motion}

In three subjects, a low spatial frequency sinusoidal grating drifting through a circular aperture (Fig. 1a) at moderate speed elicits vigorous ocular following responses (Fig. 1b) similar to those previously described (Gellman et al., 1990). Figure $1 c$ plots the changes in horizontal and vertical eye positions over a 95-135 msec time window, as a function of grating motion direction. Continuous lines are best-fitting sine functions computed to evaluate the peak-to-peak amplitude modulation throughout the complete range of motion direction. As expected, the changes in horizontal and vertical eye position are well fitted by cosine and sine functions, respectively, with amplitude ranging from 0.103 to $0.031^{\circ}$ (mean \pm SD across subjects; horizontal: $0.075 \pm 0.028$; vertical: $\left.0.063 \pm 0.028^{\circ}\right)$.

Latencies of ocular following responses were measured for each subject and each grating motion direction (Fig. 1d). For motion along the cardinal axes, latencies can be measured only for either horizontal or vertical eye movements. Across subjects, mean ( \pm SD across trials) latencies ranged between $78 \pm 9$ and $86 \pm 8 \mathrm{msec}$. For motion along the oblique axes, latencies can be measured for both horizontal and vertical ocular responses, for each trial. Across stimulus directions and subjects, mean $( \pm$ SD across trials) latencies of vertical and horizontal ocular responses ranged from $75 \pm 8$ to $87 \pm 7$ msec. On a trial-by-trial basis, we computed the absolute latency difference between vertical and horizontal responses. Averaged across directions, latency differences were $3 \pm 1,5 \pm 2$, and $6 \pm 3 \mathrm{msec}$, for subjects G.M., I.B., and Y.R., respectively. Across subjects, grand average $( \pm \mathrm{SD})$ latency difference ranged between $2 \pm 13$ and $8 \pm 11 \mathrm{msec}$. An ANOVA conducted over the four oblique motion conditions indicated that there was no effect of motion direction on either vertical or horizontal response latencies and no significant difference between latency measurements.

\section{Responses to type I plaid motion}

Figure 2a illustrates one frame of a type I plaid pattern obtained by summing two moving gratings, with a $90^{\circ}$ orientation difference. Eight possible pairs were chosen to produce eight different pattern motion directions, $45^{\circ}$ spaced. Component directions were always $+45^{\circ}$ and $-45^{\circ}$ relative to plaid motion direction. Drifting plaids elicited ocular following responses almost identical to those observed with single gratings (compare Figs. $1 b, 2 b$, same naive subject). Figure $2 c$ plots the horizontal and vertical changes in position over the $95-135 \mathrm{msec}$ time window, as a function of pattern motion direction. Best-fitting sine functions were almost identical to those obtained for single grating motion, and no significant differences were observed between parameters estimated with either grating or plaid stimuli. Note that, peak-topeak amplitudes of the cosine and sine functions were almost identical (mean across subjects, horizontal: $0.077 \pm 0.016$; vertical: $0.072 \pm 0.026$; comparison with grating-driven responses, unpaired Student's $t$ test, $p>0.4)$. Figure $2 d$ plots the mean ( $\pm \mathrm{SD}$ ) latencies of horizontal (white bars) or vertical (black bars) eye movements, for each subject and each plaid motion direction. 

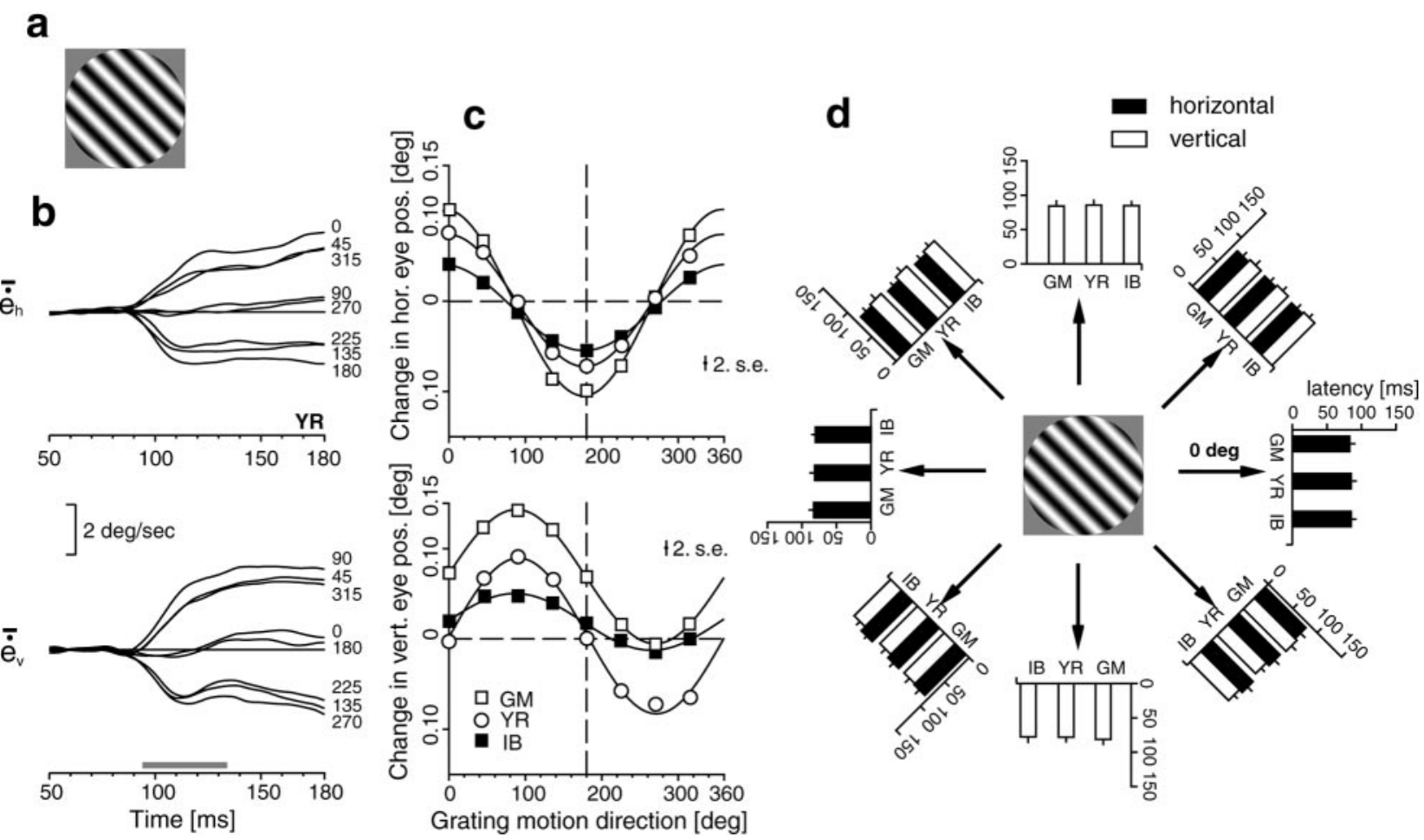

Figure 1. Ocular following responses to grating motion. $a$, One frame of the low spatial frequency grating seen through a $20^{\circ}$ diameter circular aperture. $b$, Mean horizontal $\left(\overline{\mathrm{e}}_{\mathrm{h}}\right)$ and vertical ( $\left.\overline{\mathrm{e}}_{\mathrm{v}}\right)$ eye velocity profiles to eight different grating motion directions, indicated by right-hand numbers. $c$, Individual mean changes in horizontal (top plot) and vertical (bottom plot) positions, over a 95-135 msec time window ( gray bar, plots b), as a function of grating motion direction. Continuous lines are best-fitting sine functions. $d$, Latency of ocular following responses. For each grating motion direction, white and black bars indicate mean $( \pm \mathrm{SD})$ latency of horizontal and vertical eye movements, respectively, for each subject. Notice that for horizontal (vertical) grating motion, latencies can be measured only for the horizontal (vertical) eye movements.

Mean latencies ( \pm SD, across three observers) of horizontal and vertical eye movements were $86 \pm 3.8$ and $88 \pm 8 \mathrm{msec}$ and were not significantly different from the latencies observed with grating motion.

For each trial we computed the earliest (95-135 msec time window) tracking direction from horizontal and vertical changes in position. Figure $3 a$ plots the distribution frequencies, for all three subjects, of tracking responses to either a single grating (broken lines) or a type I plaid pattern, both moving upward. It is evident that both types of motion stimuli triggered responses in approximately the correct direction, with similar variances and biases. This similarity is further illustrated by plots in Figure $3 b$ in which mean values of the distribution obtained with grating or plaid motion are plotted against one another, for all motion directions. For all three subjects, significant linear relationships of slopes $\sim 1$ (mean \pm SD across subjects: $0.94 \pm 0.04, r^{2}>0.978$ ) were found, indicating that very little difference could be noticed for tracking accuracy of ocular following to plaid and grating stimuli.

It has been suggested that the initiation of pursuit eye movement to two single-spot targets (Lisberger and Ferrera, 1997) or two random-dot patterns (Mestre and Masson, 1997) moving simultaneously can be predicted from the vector average of the ocular responses observed with each target, presented independently. We asked whether the $2 \mathrm{D}$ tracking response to plaid stimuli can be predicted from a weighted sum of the tracking responses to component gratings presented independently, because a similar linear computation would be sufficient to extract the perceived direction of a type I "symmetrical" plaid (Adelson and Movshon, 1982). This hypothesis is further supported by the fact that, for a given stimulus motion direction, plaid-driven and grating-driven responses were of similar accuracy (Fig. 3b) and amplitude. The latter point is illustrated by Figure $4 a$ for subject Y.R. where changes in horizontal or vertical positions observed with either plaid or grating motion are plotted against one another. These relationships were fit by linear regression functions of slopes 1.1 and 1.15, respectively (intercepts: -0.0002 and $\left.-0.0013 ; r^{2}>0.997\right)$. Similar linear relationships were found for the other subjects, with slopes ranging from 0.85 to 1.54 (mean \pm SD across observers, $1.06 \pm 0.18$ and $1.2 \pm 0.32$ for horizontal and vertical changes in position, respectively; $\left.r^{2}>0.986\right)$.

Figure $4 b$ plots the frequency distribution of initial tracking responses driven by either a type I plaid (continuous line) moving in the oblique $\left(+45^{\circ}\right)$ direction or by a single grating (dashed lines) moving along either the horizontal $\left(0^{\circ}\right)$ or the vertical $\left(90^{\circ}\right)$ meridian. It can be seen that responses were always initiated in the direction close to the motion direction, and the variance of responses was similar across conditions. The responses to each grating motion can be summarized by a mean integrated velocity vector. On a trial-by-trial basis, the $2 \mathrm{D}$ integrated velocity vectors of plaid-driven responses were decomposed into a weighted vector sum of the mean vectors of the responses driven by the component motions. Figure $4 c$ plots the results of this decomposition for the same example as in Figure $4 b$. The scatterplot shows the weight pairs computed for each trial. Pairs were broadly distributed around the mean weights of $(0.8 ; 0.78)$ (black dot). The histograms plot the frequency distribution for each weight. They show that distributions were unimodal, ruling out the hypothesis that, on some trials, responses to plaid motion can be 

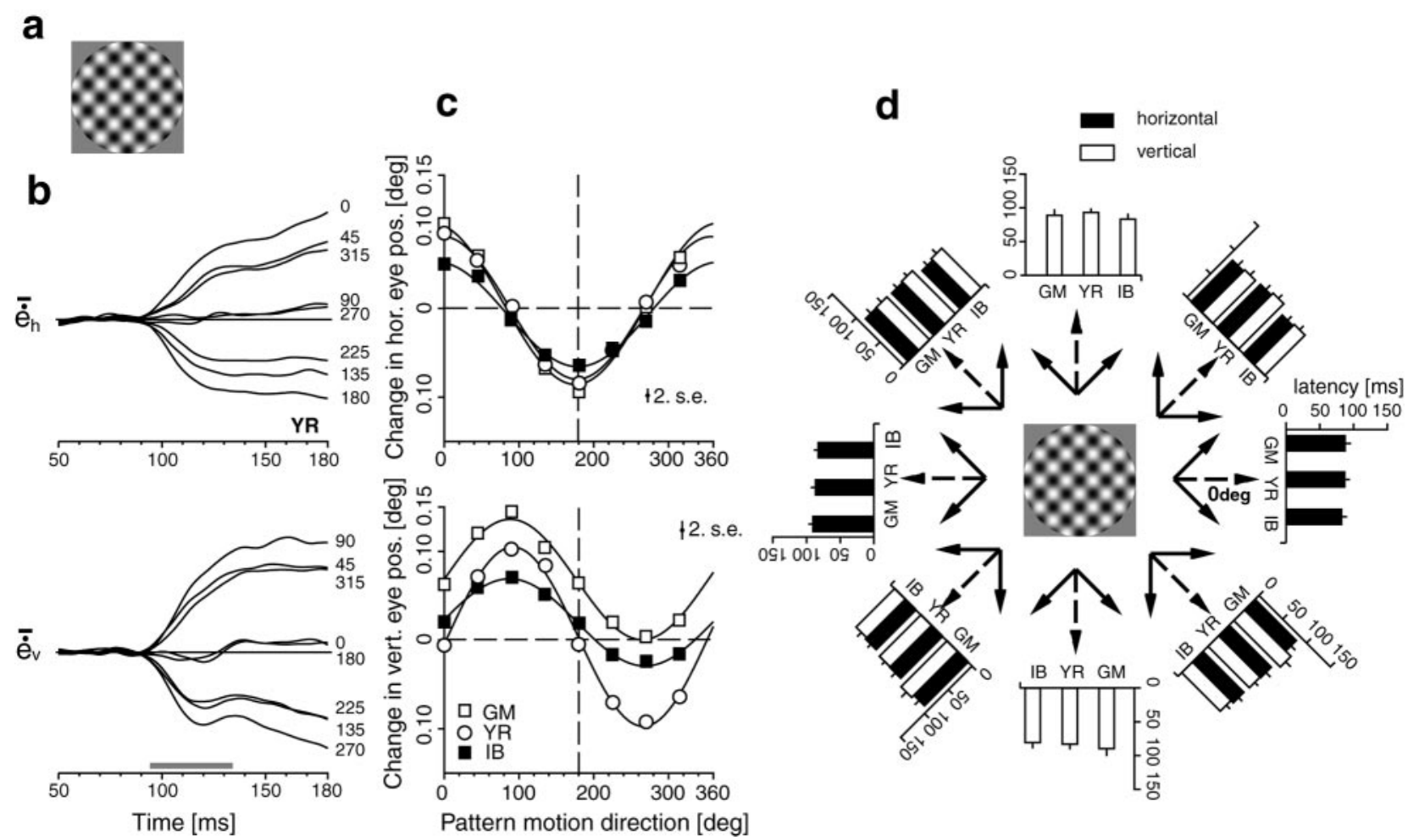

Figure 2. Ocular following responses to type I plaid motion. $a$, One frame of a low spatial frequency plaid pattern seen through a $20^{\circ}$ diameter circular aperture. $b$, Mean horizontal ( $\left.\tilde{\mathrm{e}}_{\mathrm{h}}\right)$ and vertical ( $\left.\tilde{\mathrm{e}}_{\mathrm{v}}\right)$ eye velocity profiles to eight different pattern motion directions, indicated by right-hand numbers. $c$, Individual mean changes in horizontal and vertical position, over a 95-135 msec time window ( gray bar, plots b), as a function of pattern direction. $d$, Latency of ocular following responses. For each plaid motion direction (broken arrow), white and black bars indicate mean ( \pm SD) latency of horizontal and vertical eye movements, respectively, for each subject. Notice that for horizontal (vertical) plaid motion, latencies can be measured only for the horizontal (vertical) eye movements.
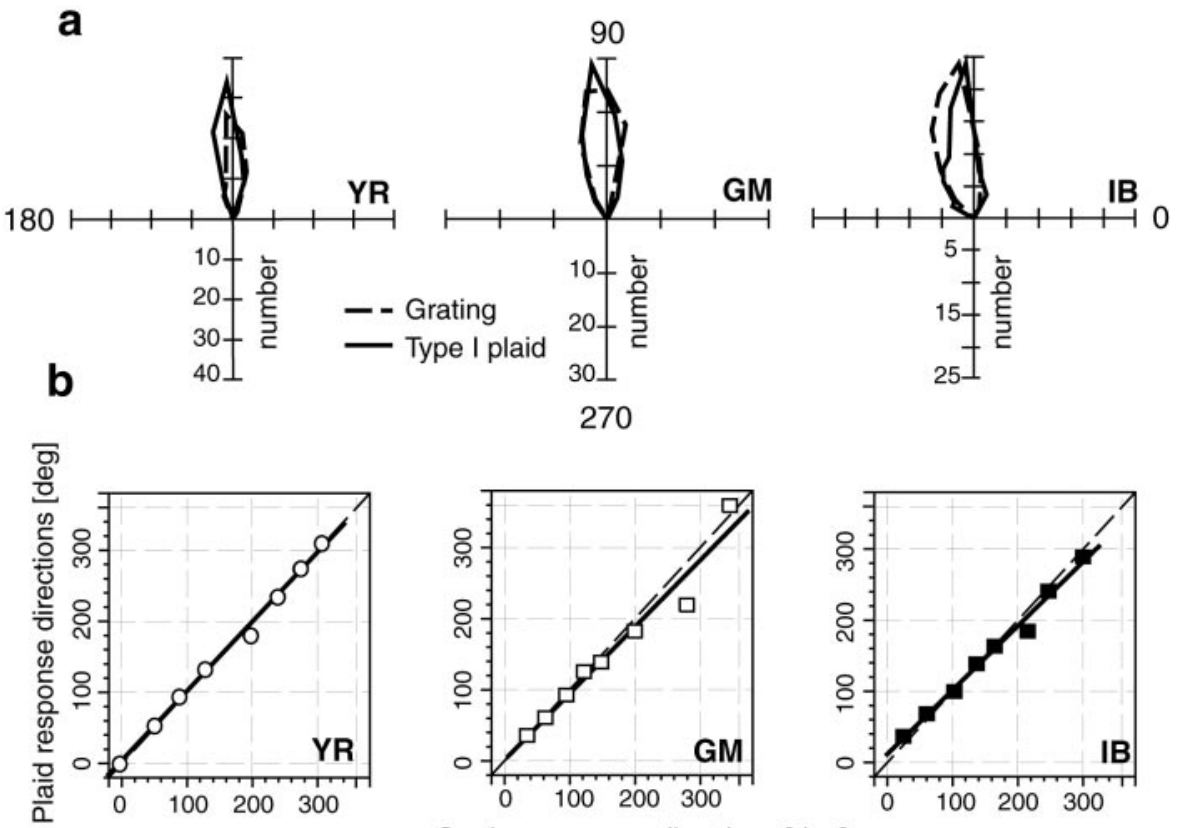

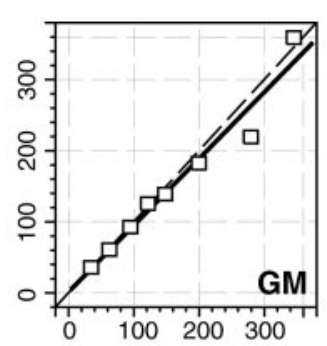

Grating response directions [deg]

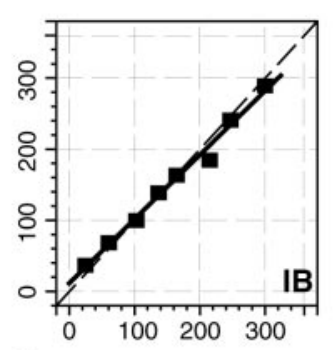

IB explained only by the tracking response to one of the components. A similar computation was performed for each pattern motion direction and each subject. Distributions were always unimodal, and mean weights ranged between 0.5 and 1.5 (mean \pm SD across subjects: $0.77 \pm 0.16)$. Across pattern directions, for each subject, mean $( \pm \mathrm{SD})$ weight pairs were of $(0.59 \pm 0.15 ; 0.60 \pm 0.21)$,
$(0.83 \pm 0.08 ; 0.79 \pm 0.07)$, and $(0.96 \pm 0.4 ; 0.90 \pm 0.36)$ for subjects G.M., Y.R., and I.B., respectively.

\section{Responses to micropatterns}

In plaid patterns, the two component gratings are superimposed. There are also 2D features ("blobs") present at the intersection of 
a

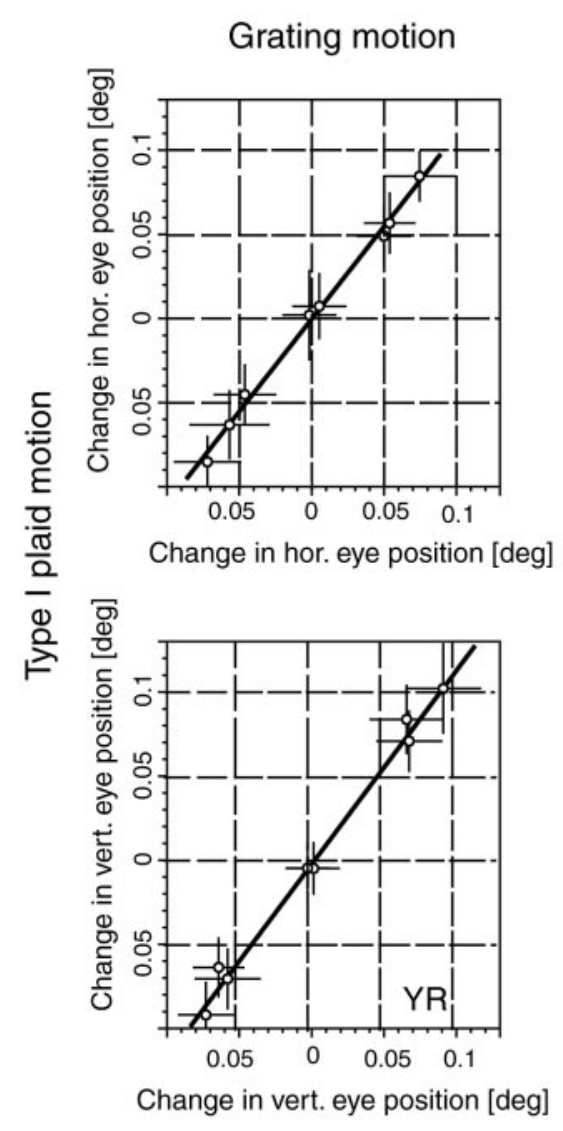

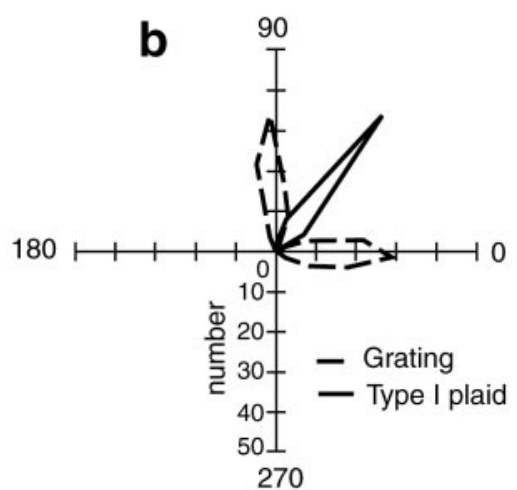
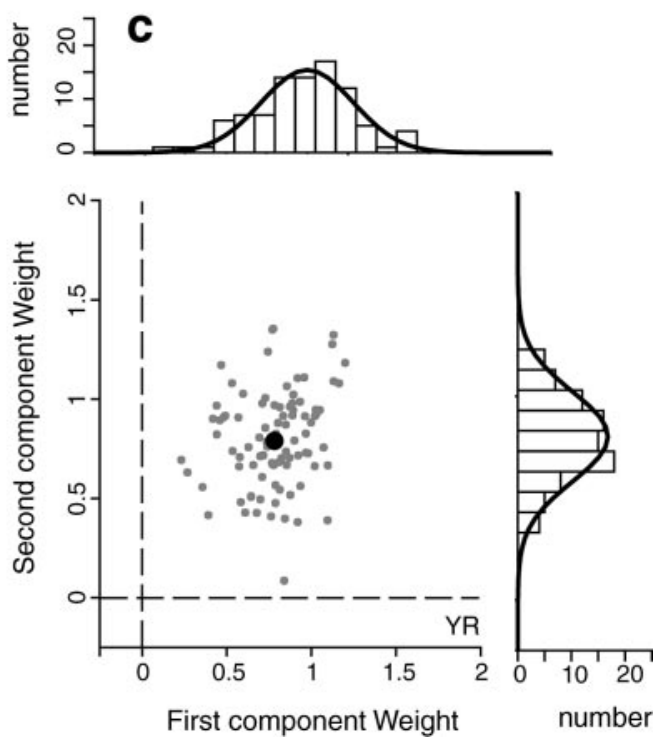

Figure 4. Relationship between grating- and plaid-driven responses. $a$, For each stimulus motion direction, mean $( \pm \mathrm{SD})$ change in horizontal (top plot) and vertical (bottom plot) positions for grating and plaid conditions are plotted one against each other. $b$, For each trial, the $2 \mathrm{D}$ response vector to a type I plaid moving along the $+45^{\circ}$ direction is decomposed as the weighted sum of the mean $2 \mathrm{D}$ response vectors observed with horizontal $\left(0^{\circ}\right)$ and vertical $\left(90^{\circ}\right)$ grating motions. Frequency histograms of first and second component weights are plotted together with the best-fit Gaussian function to indicate that weights follow unimodal distributions. Weight pairs are also plotted on a scatterplot, with mean weight pairs indicated by the black dot.c Polar plot of frequency distributions of 2D tracking directions for responses to a moving plaid (continuous lines) or to its grating components (broken lines). the two gratings, which move in the same direction as the vector average of grating motions. Ocular following responses might then be driven by these features and not by a simple linear combination of grating motions. To demonstrate that the earliest part of ocular following responses was indeed driven by a vector combination of the grating motions, we recorded ocular responses to micropattern arrays in which two Fourier motions of different directions are nonoverlapping. Figure $5 a$ illustrates one frame of either a monokinetic or a bikinetic micropattern array. In the first stimulus, carrier motion within the 16 Gabor patches were all in the same direction and therefore reproduced our previous grating motion condition. In the second stimulus, half of the carrier motions were in one direction (e.g., $0^{\circ}$ ), whereas the other half moved in another, orthogonal direction (e.g., 90 $)$. The spaceaverage direction of this micropattern array was along the oblique axis (e.g., $45^{\circ}$ ). Bottom panels illustrate the horizontal and vertical mean velocity profiles of ocular following responses to either monokinetic (Fig. 5b) or bikinetic (Fig. 5c) stimuli, for eight different global pattern motion directions. Both patterns elicited tracking responses with the usual ultra-short latency ( $\sim 85 \mathrm{msec})$. Direct comparison between Figure $5, b$ and $c$, indicates that responses were very similar for a given global motion direction, regardless of the type of stimulus. Changes in either horizontal or vertical eye position over a $95-135 \mathrm{msec}$ time window were fit with cosine and sine functions, respectively. Best-fit parameters were almost identical for unikinetic and bikinetic motions, indicating that no significant differences were seen between mean responses to either type of micropattern stimuli. This is further illustrated in Figure $5 d$. For the same subject (Y.R.), the mean $( \pm \mathrm{SD})$ changes in horizontal and vertical positions for either monokinetic or bikinetic patterns are plotted against one another. A linear regression fit the two sets of data very well $\left(r^{2}>0.991\right)$ with slopes of 0.87 and 0.92 for horizontal and vertical eye movements, respectively. Similar linear relationships $\left(r^{2}>0.981\right)$ were found for three other subjects with slopes ranging from 0.78 to 1.05 (mean \pm SD across observers: $0.92 \pm 0.1$ and $0.91 \pm 0.09$ for horizontal and vertical positions, respectively).

The same vector decomposition as performed above for type I plaids was computed to see if responses to a bikinetic pattern were predicted from the responses to its unikinetic component patterns. Data are plotted in the same way as Figure 5, with pattern directions of 0 and $90^{\circ}$ (unikinetic) or $+45^{\circ}$ (bikinetic). Again, it can be seen that frequency distributions of initial tracking directions (Fig. 6b) were centered about the global motion direction, with both types of patterns. Notice that, with a stimulus made of two different carrier motion directions, ocular following responses are initiated in the direction of the global motion, which is not physically present at any one spatial location in the stimulus. These responses can be decomposed as a weighted sum of the responses to the corresponding unikinetic micropattern arrays. Figure $6 b$ plots the frequency distribution of first and second weights (across trials) and the scatterplot of each trial-by-trial weight pair. Again, distributions were always unimodal. For this particular condition, the mean weight pair was $(0.67 ; 0.64)$. Across 
a
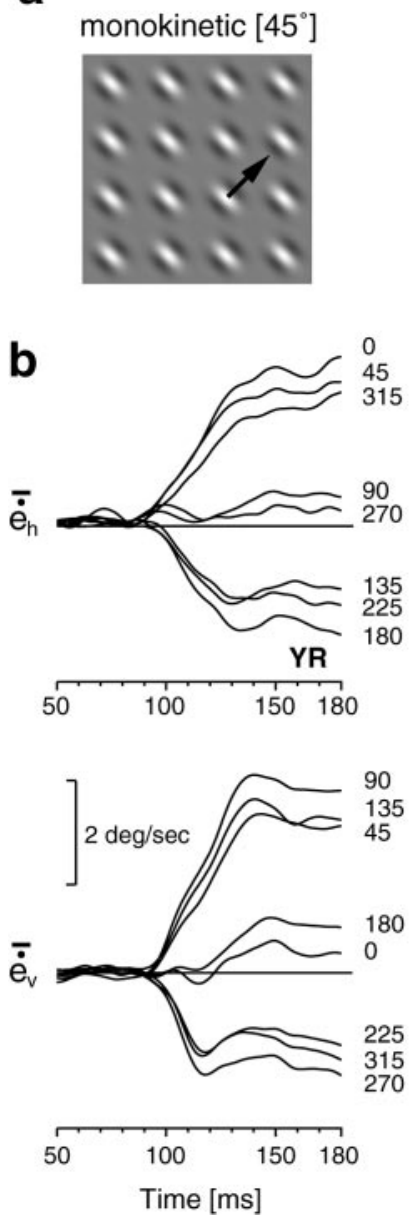

bikinetic $\left[0^{\circ}, 90^{\circ}\right]$
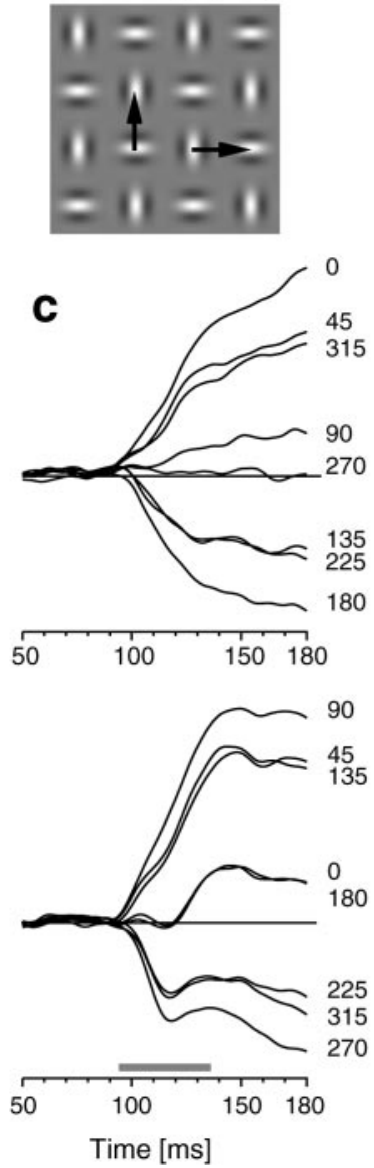

d
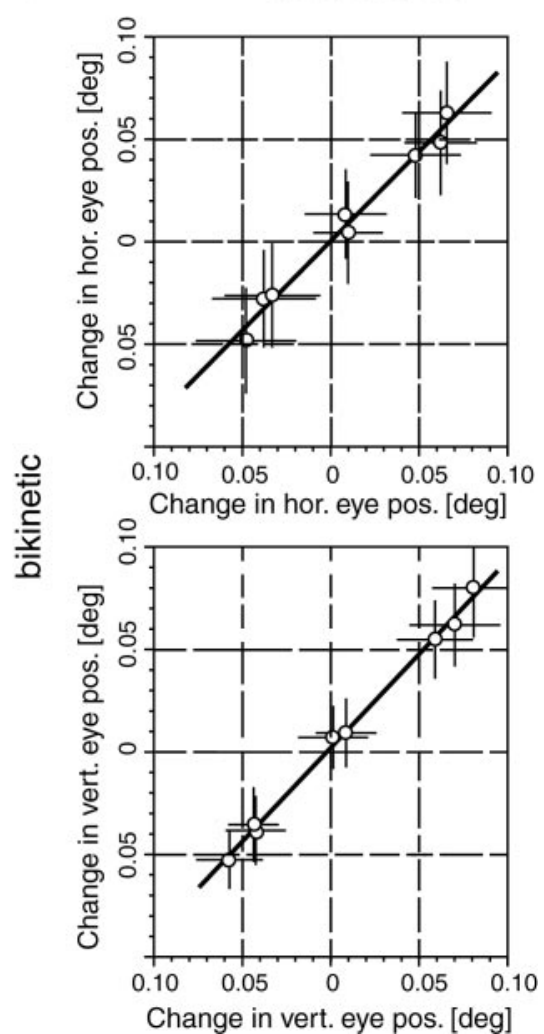

Figure 5. Ocular following responses to micropatterns. $a$, One frame of a monokinetic or a bikinetic micropattern made of 16 Gabor patches. Global motion directions are identical for both patterns. $b$, Mean horizontal $\left(\overline{\mathrm{e}}_{\mathrm{h}}\right)$ and vertical $\left(\overline{\mathrm{e}}_{\mathrm{v}}\right)$ eye velocity profiles to eight different monokinetic pattern motion directions, indicated by right-hand numbers. Compare with the ocular following responses observed with bikinetic patterns moving in the same global motion directions $(c)$. $d$, Mean $( \pm \mathrm{SD}$ ) changes in horizontal (top plot) or vertical (bottom plot) directions observed with either monokinetic or bikinetic patterns are plotted against each other.

subjects and global motion directions, mean weights ranged between 0.31 and 1.45 (mean \pm SD across subjects: $0.64 \pm 0.12$ ). Mean weights across subjects were not significantly different from those observed for type I plaid (unpaired $t$ test, $p=0.32$ ). Across pattern directions, for each subject, mean $( \pm \mathrm{SD})$ weight pairs were $(0.5 \pm 0.1 ; 0.52 \pm 0.05),(0.65 \pm 0.1 ; 0.64 \pm 0.05)$, and $(0.72 \pm 0.22 ; 0.82 \pm 0.34)$ for subjects G.M., Y.R., and I.B., respectively.

\section{Responses to type II plaid motion}

We recorded the ocular following to "unikinetic" type II plaids where grating and pattern motions have different directions. Plaids were constructed by summing a stationary and a moving grating of the same spatial frequency but with a $45^{\circ}$ orientation difference. Figure $7 b$ plots for three subjects, the mean horizontal and vertical velocity profiles of the ocular responses obtained with one subset of conditions that are illustrated in Figure $7 a$. An oblique stationary grating was added to an horizontal grating that drifted either upward or downward. With an upward moving grating, pattern motion was upward and rightward (broken arrow, 1 ). With a downward moving grating, pattern motion was downward and leftward (broken arrow, 2). Grating and plaid motion stimuli were fully interleaved, together with other pairs of grating orientations. Tracking responses to plaids exhibited the striking properties illustrated in Figure $7 b$. For all three subjects, vertical grating motion elicited vigorous responses in the vertical direction (bottom plots, continuous lines), at ultrashort latencies. With moving plaids, very similar vertical responses were again elicited, at short-latency (bottom plots, broken lines). However, a second component was seen in the horizontal velocity profiles (top plots, broken lines). Its direction depended on the moving pattern: rightward with pattern 1 , leftward with pattern 2 . This second component was largely delayed relative to the vertical tracking responses. In all three subjects, horizontal eye movements were initiated with latencies longer than $100 \mathrm{msec}$, as indicated by the second vertical dotted lines. For each trial, we measured the horizontal and vertical latencies and then computed the latency difference $(\delta)$ between the early and late tracking components.

Figure 8 plots the mean ( \pm SD across trials) horizontal and vertical latencies for three subjects, in a complete subset of conditions in which the static grating was always oblique (Fig. 7a), and was combined with three different gratings moving in six different directions. Notice that there were two instances where grating and plaid motions had the same directions because the orientation difference between static and drifting grating was $90^{\circ}$. 

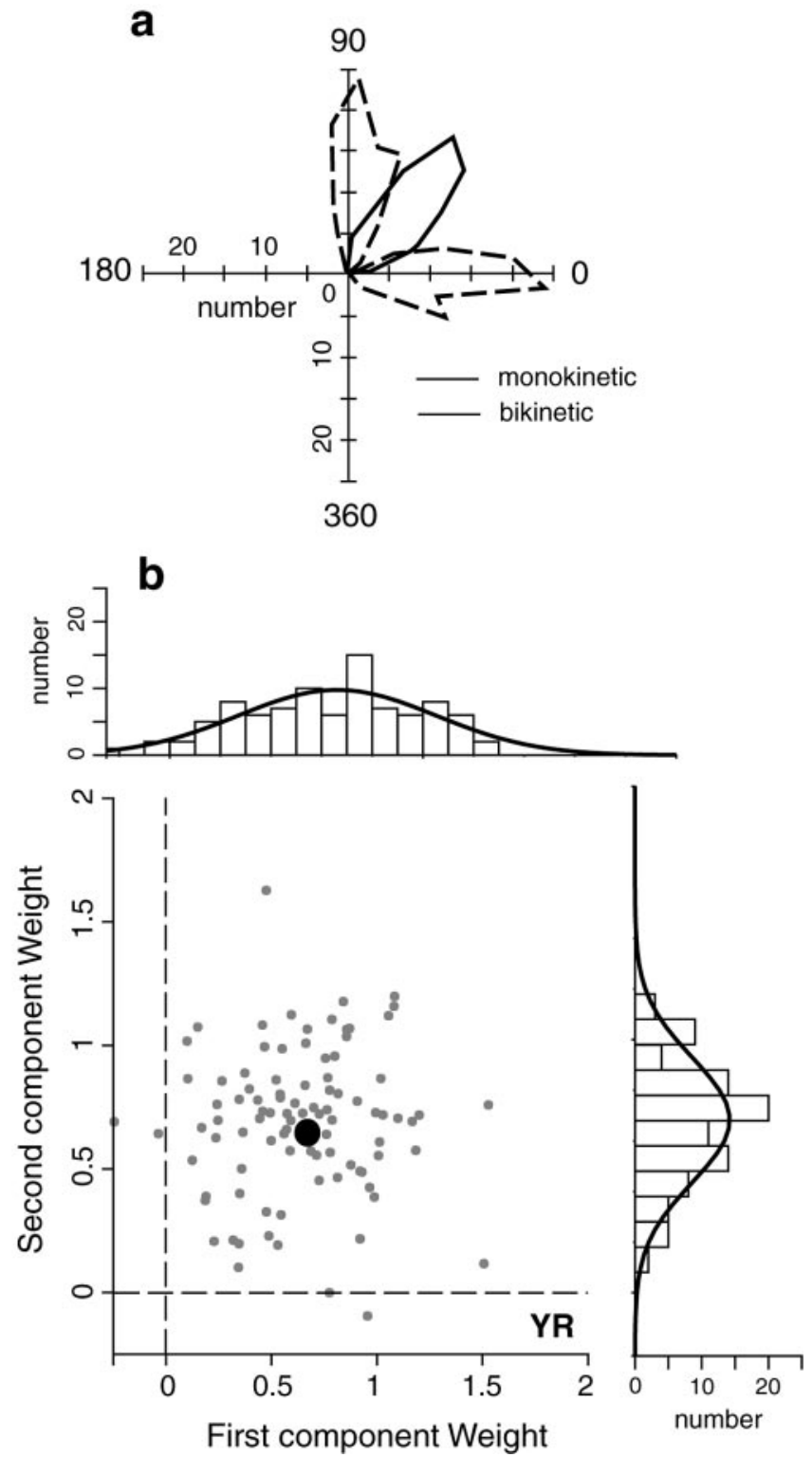

Figure 6. Vector decomposition of responses to bikinetic patterns. The analysis of $2 \mathrm{D}$ vectors of tracking responses is shown for one subject (Y.R.) when presented with either monokinetic (rightward or upward carrier motions) or bikinetic (rightward and upward carrier motions) micropatterns. $a$, First and second component weights are plotted against each other, for each trial. Black dot indicates the mean weight pair. The frequency distributions of each weight are also plotted as histograms $(b$, $c$ ) together with best-fit Gaussian functions. $d$, Polar plot of the frequency distribution for the 2D tracking direction of responses to monokinetic (broken lines) or bikinetic patterns.

These latter conditions enabled us to investigate whether the presence per se of a static grating had any detrimental effect on the earliest ocular following to grating motion. For all subjects, under such circumstances tracking was initiated in the oblique directions at the shortest latency, as indicated by similar latencies of both horizontal and vertical eye movements (mean \pm SD across subjects: $87 \pm 6.8$ and $87 \pm 8.7 \mathrm{msec}$, respectively). Latency differences were computed for each trial and were negligible (mean \pm SD across conditions: $3 \pm 1.5,3 \pm 2.8$, and $2 \pm 0.5$ msec for subjects G.M., Y.R., and I.B., respectively).

In the four other conditions, grating motion was along cardinal axes, whereas pattern motion was along the oblique axis. As a consequence, grating- and pattern-driven responses can be identified from the vertical and horizontal eye movements. For instance, when a vertical component grating was drifted either rightward or leftward, latencies of horizontal eye movements (black bars) were found to be shorter than latencies of vertical eye movements (white bars). From Figure 8, it can be seen that, for all these conditions, an early and late component were always present, for all subjects. Latencies of the early component ranged from $78 \pm 4$ to $93 \pm 4$ msec (mean \pm SD across directions, for each observers). Latencies of the later component ranged from $99 \pm 3$ to $118 \pm 8$. For each condition and each subject, the latency difference between early and late component was highly significant (unpaired $t$ test, $p<0.00001$ ). For each trial, the latency difference $(\delta)$ between horizontal and vertical eye movements was computed. Across conditions, mean $\delta$ values were $29 \pm$ 8 (G.M.), $25 \pm 5$ (Y.R.), and $21 \pm 2 \mathrm{msec}$ (I.B.). Across subjects and directions, mean $\delta$ value was $25 \pm 4 \mathrm{msec}$ for those conditions in which grating and pattern motions had different directions. Similar results were observed with a grating tilted along the other oblique axis $\left(-45^{\circ}\right)$, where mean $\delta$ value ( \pm SD across observers) was $18 \pm 3 \mathrm{msec}$.

The later component guided ocular following toward the pattern motion direction. This is further illustrated by computing on a millisecond-by-millisecond basis the mean $2 \mathrm{D}$ velocity vector of responses to either grating or type II plaid motion. Figure 9 illustrates for the three subjects, these instantaneous velocity vectors (plotted only every $4 \mathrm{msec}$, for clarity), as a function of time for a horizontal grating moving upward, presented either alone or with an oblique static grating. For pure grating motion, responses were initiated immediately in the upward $\left(+90^{\circ}\right)$ direction and stayed approximately aligned with stimulus direction for the stimulus duration. In contrast, responses to a moving plaid were first initiated in the upward direction but then slowly deviated toward the pattern motion direction $\left(+45^{\circ}\right)$, with different time courses for each subject. We computed the integrated velocity vector of the tracking responses over the 135-175 msec time windows, for each trial. Left-hand plots in Figure 9 are the frequency distributions of the $2 \mathrm{D}$ vector directions. They show that at the end of a trial, ocular following responses were primarily deflected toward the pattern motion direction, with little variance. For the examples shown in Figure 9, mean final tracking directions were $81^{\circ}$ (Y.R.), $89^{\circ}$ (G.M.), and $91^{\circ}$ (I.B.) for $+90^{\circ}$ grating motion and $56^{\circ}$ (Y.R.), $67^{\circ}$ (G.M.), and $69^{\circ}$ (I.B.) for a $+45^{\circ}$ pattern motion.

To compare the initial tracking directions observed with gratings and unikinetic plaids, we plotted the mean $( \pm \mathrm{SD}$ across subjects) direction observed in response to single gratings (Fig. $10 a$ ) or unikinetic plaids with four different static grating orientations (Fig. 10b-e), as a function of the grating motion direction. Broken lines indicate the predicted tracking direction if eye movements were driven by grating motion only. Continuous lines show the predicted tracking directions if ocular responses were driven by pattern motion only. As expected, ocular tracking directions were better predicted by pattern motion than by grating motion, for all static grating orientations. Mean errors $( \pm \mathrm{SD}$ across grating motion directions) between tracking and pattern motion directions were $28.9 \pm 25^{\circ}, 25.1 \pm 15^{\circ}, 21.4 \pm 14^{\circ}$, and $23.6 \pm 8.8^{\circ}$ for static grating orientations of $0,+45,-45$, and $+90^{\circ}$, respectively. This indicates that, over the $135-175 \mathrm{msec}$ time window, tracking direction was displaced halfway between grating and pattern motion directions. 

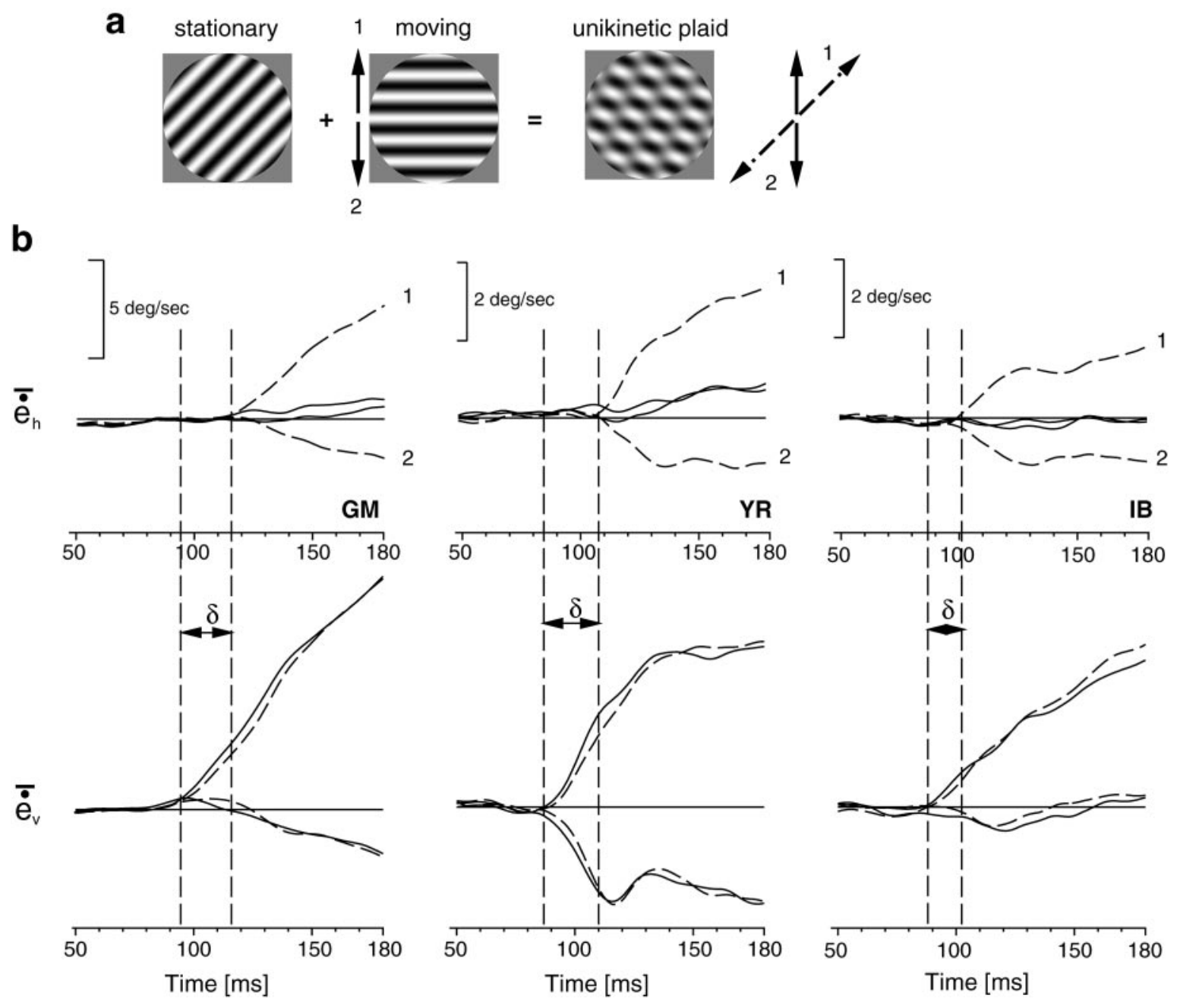

Figure 7. Tracking responses to type II unikinetic plaid motion. $a$, Two examples of motion stimuli. For upward grating motion (1), pattern moves rightward and upward; for downward grating motion (2), pattern moves leftward and downward. $b$, Horizontal ( $\left.\dot{\mathrm{e}}_{\mathrm{h}}\right)$ and vertical ( $\left.\dot{\mathrm{e}}_{\mathrm{v}}\right)$ velocity profiles for three subjects, in response to single grating motion (continuous lines) or to moving plaids (dashed lines). Vertical dotted lines plot the mean latency estimates of both horizontal and vertical eye movements to plaid motion, yielding a mean latency difference $(\delta)$ of $\sim 20 \mathrm{msec}$.

\section{Pattern motion coherency: effect of relative spatial frequency}

When the two component gratings are of very different spatial frequency, motion coherency breaks down, and subjects perceive two gratings moving independently: motion transparency (Adelson and Movshon, 1982). The effect of relative spatial frequency on pattern coherency of unikinetic plaids has been primarily overlooked by previous studies (Goréa and Lorenceau, 1991; Dobkins et al., 1998). We investigated the effect of the relative spatial frequency between component gratings on ocular following responses. To keep both grating and pattern velocity vectors constant, we fixed the spatial frequency of the moving grating at $0.27 \mathrm{cpd}$, and we varied the spatial frequency of the static grating from 0.14 to $3.2 \mathrm{cpd}$. Figure 11 shows the results for one set of conditions, where the moving grating drifted upward and the static grating was oblique so that pattern motion direction was upward and leftward (Fig. 11b). Figure 11a illustrates the mean velocity profiles, for one subject (G.M.) observed with the different spatial frequencies of the static grating. The latency of the vertical responses was $\sim 80 \mathrm{msec}$. As expected, a later component was seen in the horizontal eye movements, at latency $\sim 100 \mathrm{msec}$.
The amplitude of this later component was strongly modulated by the spatial frequency of the static grating, whereas its latency remained constant. Increasing the spatial frequency from 0.12 to $0.8 \mathrm{cpd}$ increased the initial velocity of the horizontal responses (continuous lines), although higher spatial frequencies resulted in a progressive decrease of the horizontal eye velocity (broken line). By contrast, changing the static spatial frequency had no effect on the earlier, vertical response component because all velocity profiles were superimposed.

Changes in both horizontal and vertical positions were computed for each trial over the 135-175 msec time window. Figure $11 c$ illustrates the inverted U-shaped relationship found between the change in horizontal position and the spatial frequency of the static grating, for the three subjects. For comparison, horizontal broken lines indicate, for each subject, the change in horizontal position observed with a single upward grating motion, to show the baseline response seen in the absence of $2 \mathrm{D}$ pattern motion. For both the lowest and highest spatial frequencies, the later component was almost reduced to the individual, baseline responses. For those conditions, motion coherency broke down, and subjects reported that they perceived motion transparency, a 


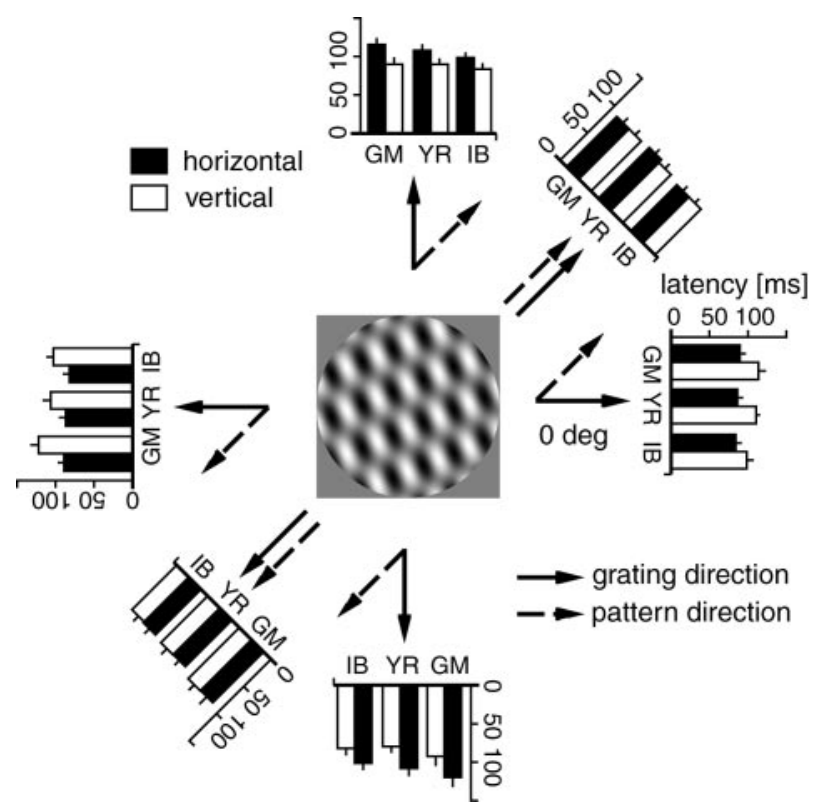

Figure 8. Latency of early and late tracking components. Mean $( \pm$ SD $)$ latency of vertical and horizontal eye movements in response to unikinetic plaids. For all conditions, the static grating is tilted $-45^{\circ}$. Six different grating motion directions are illustrated (continuous arrows), corresponding to six different pattern motion directions (broken arrows). When grating and pattern are in the same direction, no difference between vertical and horizontal latencies are observed. When grating and pattern directions differ by $45^{\circ}$, latencies are systematically shorter in the direction of the grating motion.

moving grating sliding over a static grating. Peaks of the U-shaped tuning functions were found for static spatial frequencies between 0.5 and $1 \mathrm{cpd}$. It can be seen that the overall amplitude of the later component varied from one subject to another, without any correlated change in the peak location. To give a precise estimate of peak spatial frequencies, data were normalized and fit with a double-exponential function:

$$
A=a \times \exp (b \times f)-c \times \exp (d \times f)
$$

where $A$ is the normalized amplitude of the late response, $f$ is the static spatial frequency, and $a, b, c$, and $d$ are free parameters with positive values. Figure 12 plots these normalized data for the three subjects, together with best-fit functions. Estimated peaks of the functions were located between 0.71 and $0.77 \mathrm{cpd}$, that is 2-3 times higher than the spatial frequency of the moving grating $(0.27 \mathrm{cpd}$, vertical arrow). Similar values were obtained with the three other grating motion directions [mean \pm SD across directions: $0.78 \pm 0.02$ (G.M.); $0.71 \pm 0.05$ (Y.R.), and $0.73 \pm 0.03$ (I.B.)]. Thus, on average, the largest responses in the pattern motion direction were observed when the ratio between static and moving spatial frequencies was $\sim 1.5$ octaves.

\section{Pattern motion coherency: effect of relative contrast}

Earlier studies on plaid motion perception reported that motion coherency also failed when the two gratings have very different contrast (Adelson and Movshon, 1982). Under such conditions, the two gratings did not cohere, and subjects reported seeing two gratings sliding over each other. Similar motion transparency was observed by Dobkins et al. (1998) using unikinetic plaids when static and moving gratings have different contrasts. In the last experiment, we interleaved grating and unikinetic plaid stimuli to
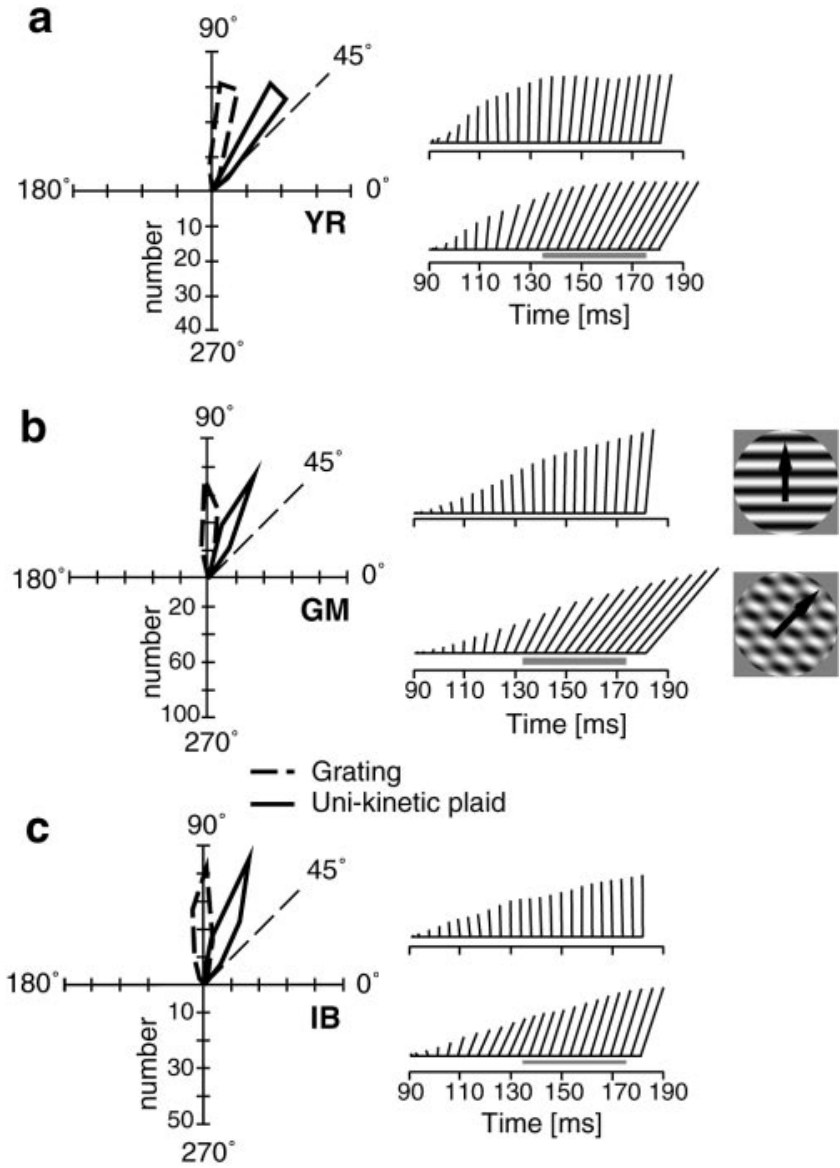

Figure 9. Tracking direction of responses to unikinetic plaids. For each subject $(a-c)$, the left panel plots the frequency distribution of 2D tracking direction for responses to either a single grating moving upward (broken lines) or a plaid pattern moving upward and rightward $\left(45^{\circ}\right)$ (continuous lines). Right panel plots the instantaneous mean velocity vector of responses to either grating or type II plaids. Vectors are computed from mean horizontal and vertical velocity profiles (see Fig. 7) and shown every $4 \mathrm{msec}$.

probe the contrast response functions of the early and late tracking components. In half of the trials, upward or downward single grating motion was presented, at contrast values ranging from 2.5 to $80 \%$. Figure $13 a$ plots the mean velocity profiles for downward single grating motion. Increasing the contrast up to $20-40 \%$ produced a sharp increase in the velocity of vertical eye movements. A change in latency was observed only for very low contrast of the moving grating. With contrast of $>40 \%$, no further increase in initial vertical eye velocity was observed. No modulation was observed for horizontal eye velocity across the whole range of moving grating contrast, indicating that cross-talk between vertical and horizontal eye movements was minimal and independent of contrast. Open symbols in Figure $13 c$ illustrate mean ( $\pm \mathrm{SE}$ ) early (95-115 msec time window) changes in horizontal (top plot) and vertical (bottom plot) positions plotted against contrast. Change in horizontal position remained approximately constant across the contrast range, whereas vertical position exhibited a steep contrast response function.

Figure $13 b$ illustrates the ocular following responses driven by a unikinetic plaid when the relative contrast between the two components was varied. Increasing the contrast of the static grating from 2.5 to $80 \%$ had no effects on the early, downward eye 
a grating alone
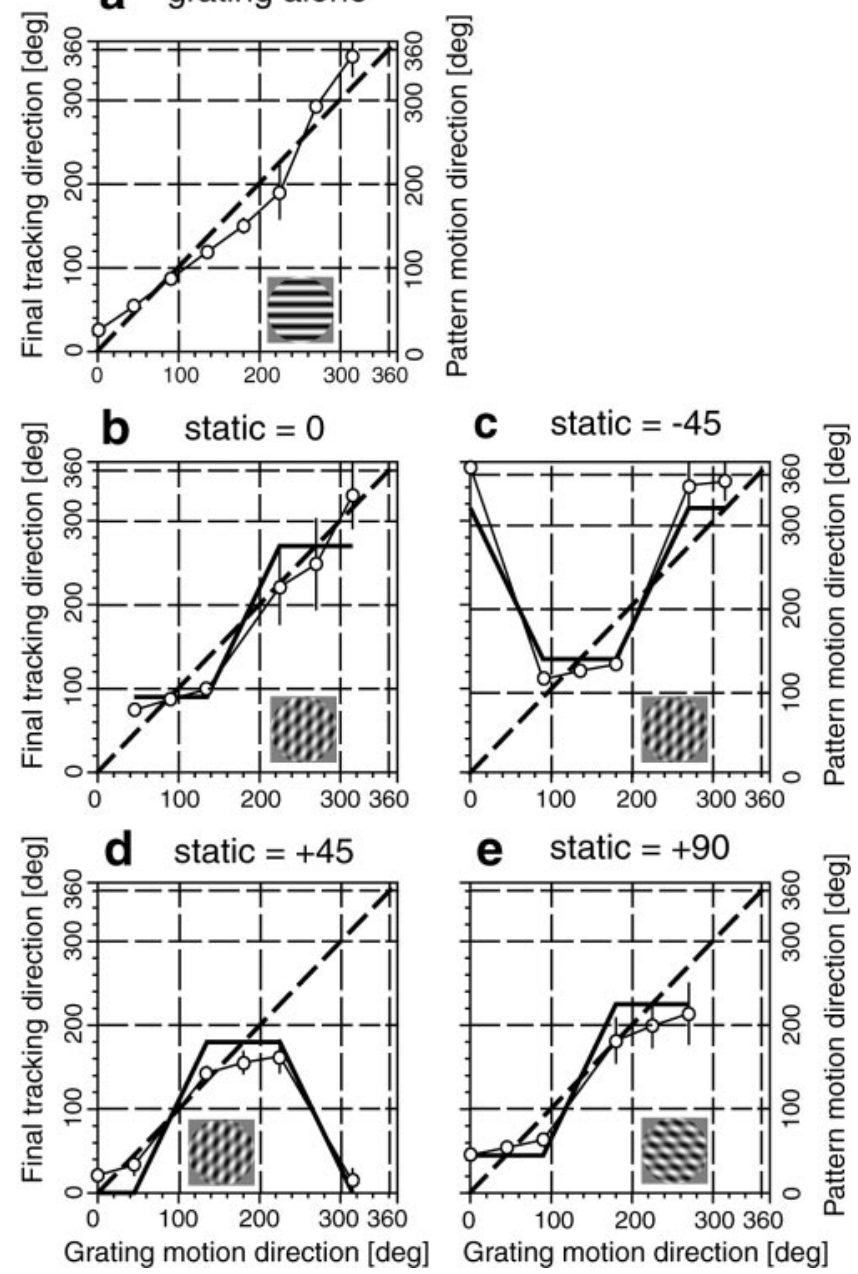

Figure 10. Predicted and observed tracking direction. Mean $( \pm$ SD across observers) final tracking directions are plotted against grating motion direction for a single grating $(a)$ or for four type II plaids with different static grating orientations $(c-e)$. Broken thick lines indicate the predicted tracking direction if ocular responses are driven solely by grating motion. Continuous thick lines indicate the predicted tracking direction if ocular responses are driven solely by pattern motion (righthand axis).

velocity, but the late, horizontal responses were strongly modulated by this static contrast. Horizontal eye velocity increased slowly as the contrast of the static grating increased from 2.5 to $60-80 \%$ with no clear saturation. These two independent effects are summarized in Figure $13 c$ (closed symbols). No modulation was seen in the vertical direction where change in vertical position over the 95-135 msec time window remained constant throughout the range of static grating contrast (bottom plot). Notice that the mean change of vertical position across the range is close to the asymptotic value obtained with a high-contrast single grating motion. On the contrary, change in horizontal position over the 135-175 msec time window (i.e., late component) exhibited a slowly increasing contrast response function. Responses were nearly negligible for static contrast of $<10 \%$. Between 10 and $80 \%$, the changes in horizontal position slowly increased with an S-shaped function.

Figure 14 plots the normalized amplitude of the early (i.e., vertical) and late (i.e., horizontal) response components, as a function of the contrast of either the moving or the static grating, respectively. We fitted the data with the Naka-Rushton function:

$$
A=c^{n} /\left(c^{n}+C^{n}{ }_{50}\right)
$$

to estimate half-saturation contrast values $\left(C_{50}\right)$ of the late and early tracking phases (Naka and Rushton, 1966). This function has been shown previously to provide a good fit of contrast response functions of neurons at various stages of the visual pathways in cats and monkeys (Albrecht and Hamilton, 1982; Sclar et al., 1990). It is however a monotonic function that cannot capture the small response decay sometimes observed with very high-contrast $(>50 \%)$ single grating (Sclar et al., 1990). In Figure 14 , continuous lines are best-fit functions, and $C_{50}$ values are indicated by vertical dotted lines. Shapes of the curves obtained for early and late tracking components were similar and can be described satisfactorily by the same function. However their sensitivities, as defined by $C_{50}$ values, were very different. For the two subjects, with plaid patterns, $C_{50}$ values of the late responses were of 34.5 and $32.02 \%$. By comparison, $C_{50}$ values for early responses observed with pure grating motion were of 6.7 and $11.8 \%$. Similar data were observed in two other naive subjects run only on this critical experiment (mean \pm SD across four observers; grating: $7 \pm 3.33 \%$ and plaid: $31.9 \pm 3.96 \%$; unpaired $t$ test, $p<0.0001)$. Similar results were found when the grating moved downward (mean \pm SD across four observers; grating: $6.09 \pm$ $3.3 \%$ and plaid: $36.33 \pm 4.74 \%$; unpaired $t$ test, $p<0.0001)$. No significant difference was observed for the exponent $n$, which determines the steepness of the contrast response functions (mean $\pm \mathrm{SD}$ across four observers; upward grating: grating: $4.35 \pm 3.7$ and plaid: $3.22 \pm 0.68$; downward grating: grating: $3.25 \pm 0.87$ and plaid: $2.93 \pm 0.78$ ).

\section{DISCUSSION}

We show that short-latency ocular following involves two kinds of motion processing that can be distinguished by their differences in both timing and contrast dependence. We found several key features of 2D motion processing: (1) pattern velocity processing is slower by $\sim 20 \mathrm{msec}$ than grating velocity processing, (2) pattern-driven responses were largest with spatial frequency differences of more that one octave, (3) grating- and pattern-driven responses have different contrast response functions, and (4) these two motion processing behave independently because varying contrast or spatial frequency of the static grating had profound effects on the late but not the early component.

\section{Short-latency ocular following responses to plaid motion}

Short presentations of moving plaids elicit vigorous, machine-like ocular following at ultrashort latencies. Consistent with previous studies, the initial tracking direction was close to the coherent motion direction of type I plaid patterns (Smith and Harris, 1991). Moreover, we show that ocular following to either a single grating, a type I plaid or a micropattern array is initiated in the global motion direction, always at the shortest latency. These early responses are best predicted by the vector average of the responses to the component motions, regardless of whether Fourier motions are overlapping (type I plaid) or not (bikinetic micropatterns). These results suggest that earliest ocular following is driven by a vector average of local Fourier motions.

Our major finding is that ocular following of unikinetic plaids reveals two components, with a latency difference of $\sim 20 \mathrm{msec}$ : an early component driven by grating motion and a late compo- 
a

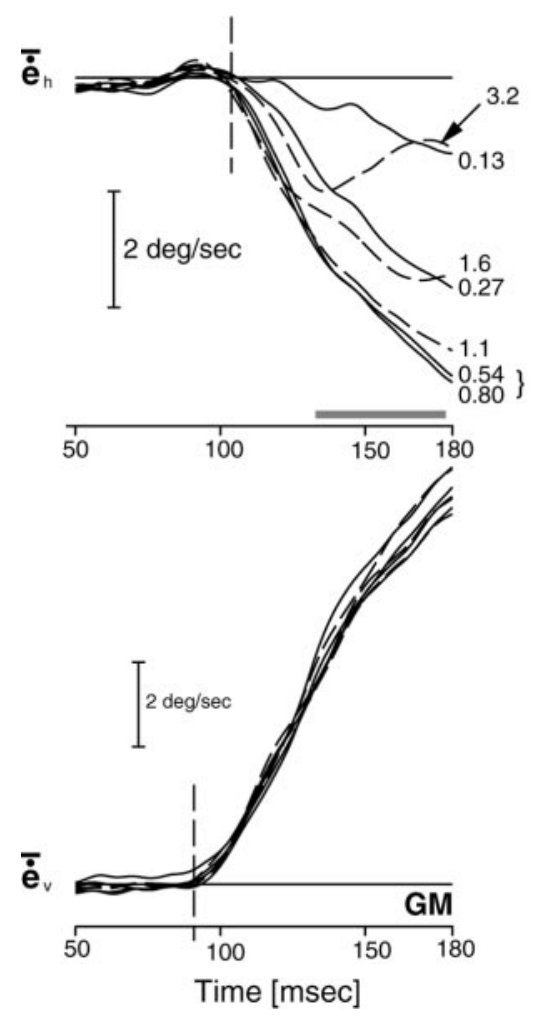

b
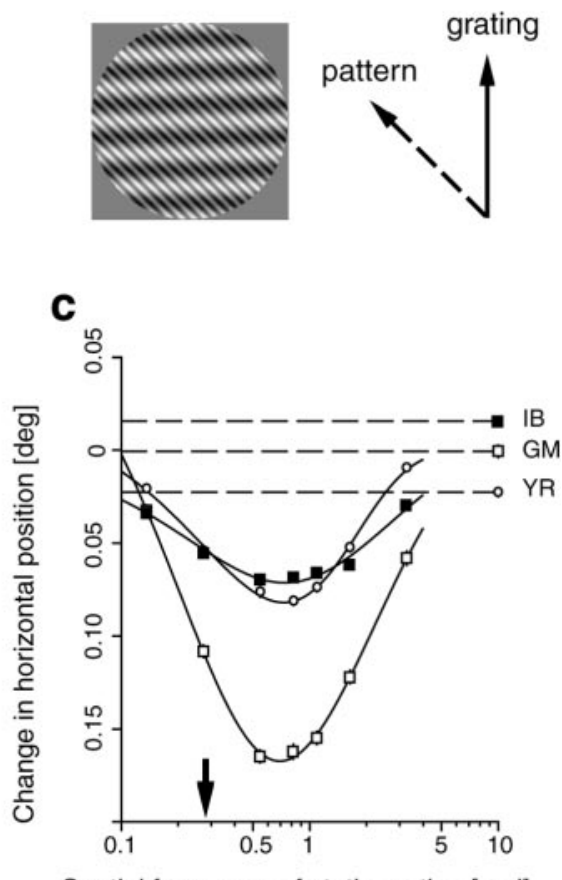

Spatial frequency of static grating [cpd]
Figure 11. Effect of relative spatial frequency. $a$, Mean horizontal and vertical velocity profiles of ocular following responses to unikinetic plaids of different static spatial frequencies (right-hand numbers). The spatial frequency of the moving grating and static grating orientation are kept constant. Grating motion is upward, and plaid pattern motion is upward and leftward (b). $c$, Change in horizontal position, as a function of static spatial frequency, for three subjects. Horizontal broken lines indicate for each subject the residual change in horizontal position observed with a pure upward grating motion. Arrow indicates the spatial frequency of the moving grating.
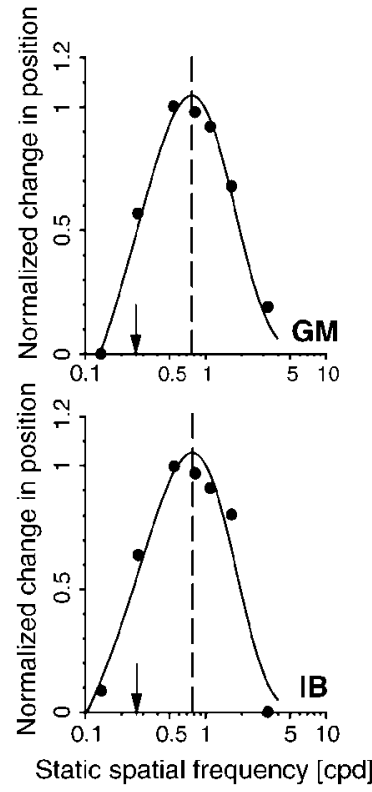

Figure 12. Relative spatial frequency tuning functions. For each subject, normalized changes in position are plotted against the spatial frequency of the static, oblique grating, for the upward grating motion condition. Continuous lines are best-fit double-exponential functions. Vertical dotted lines indicate the peak location of the tuning function. Arrows indicate the spatial frequency of the moving grating.

nent driven by pattern motion. These results are consistent with our previous finding that ocular following of "barberpole" motion stimuli (a low spatial frequency grating drifting behind an elongated, tilted aperture) is first initiated in the direction orthogonal to the grating orientation but $15-20 \mathrm{msec}$ later deviates toward the long axis of the aperture (Masson et al., 2000). Similar results were observed by Pack and Born (2001) for monkeys tracking single bars tilted $45^{\circ}$ relative to their motion axis. These results are consistent with psychophysical reports that with both elongated edges (Lorenceau et al., 1993) and plaid patterns (Yo and Wilson, 1992), short stimulus presentations yield to a perceived direction best predicted from the 1D component motions or their vector sum-average, whereas, with long stimulus presentations, the true object-pattern 2D motion direction is perceived.

\section{Tracking and 2D motion integration}

Different solutions have been proposed to solve the computational problem of reconstructing 2D pattern motion from its $1 \mathrm{D}$ component motions (Adelson and Movshon, 1982; Heeger, 1987; Wilson et al., 1992). The 1D motion of a grating is ambiguous and consistent with a family of velocities lying along a constraint line in the velocity space. Geometrically, the velocity vector of a plaid pattern can be reconstructed using the intersections of the constraint lines of each component grating : the IOC rule (Fennema and Thompson, 1979; Adelson and Movshon, 1982). An alternative approach suggests that the visual system extracts both Fourier (i.e., grating) and non-Fourier (i.e., texture pattern) signals from the moving pattern (Wilson et al., 1992; Wilson, 1999). Psychophysical (Ferrera and Wilson, 1990; Yo and Wilson, 1992) and computational (Wilson et al., 1992) studies suggest that these linear and nonlinear motion operations are performed by separate pathways that converge with different delays onto an integration stage that computes their vector sum-average to recover the surface $2 \mathrm{D}$ motion. This model also explains the parallel processing of first versus second-order motion cues (Chubb and Sperling, 

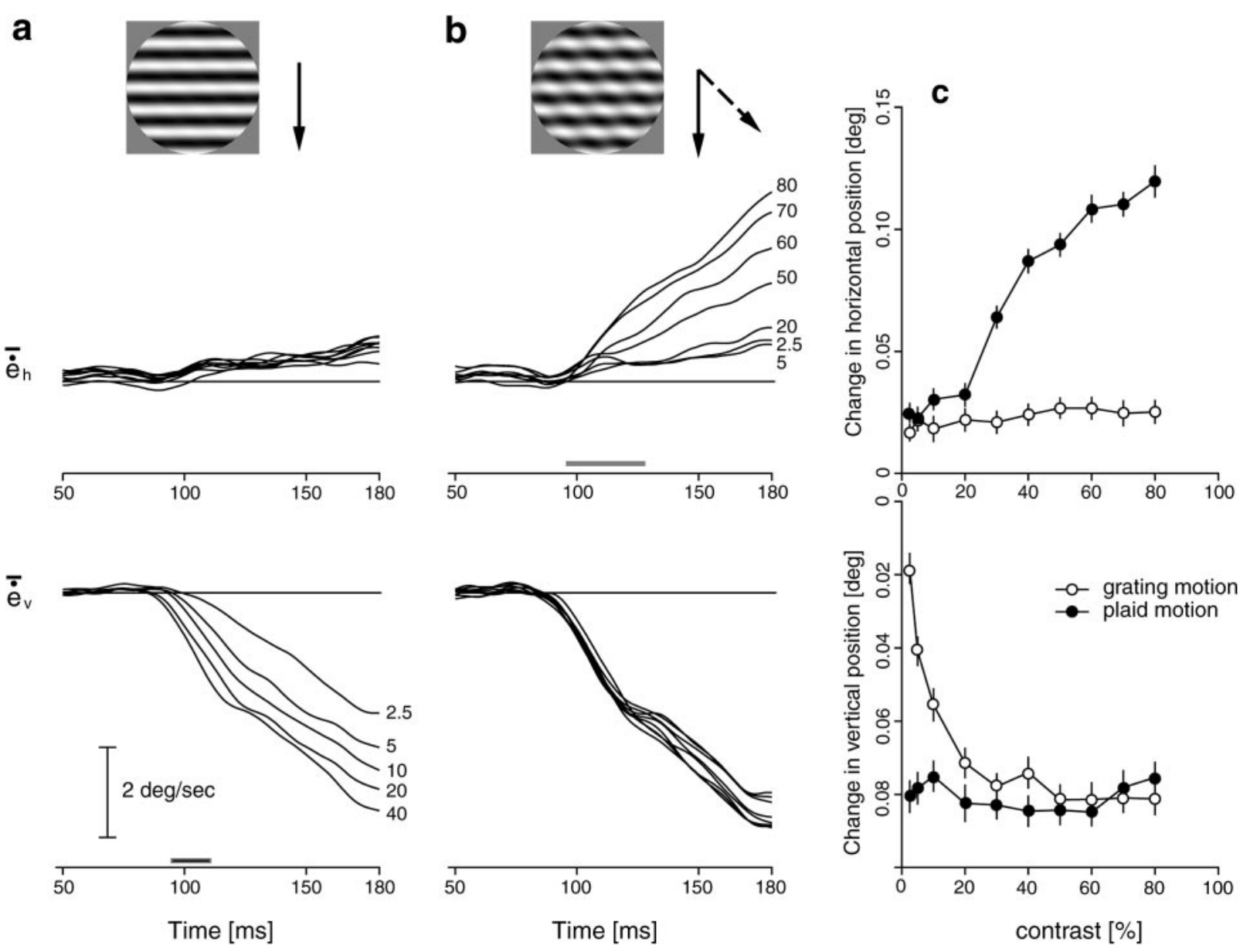

Figure 13. Effect of contrast on early and late tracking component. $a$, The contrast of a vertical grating, moving downward was varied. No changes were evident in the mean vertical eye velocity profiles, but grating contrast strongly modulated the mean vertical eye velocity. $b$, The contrast of the static grating was varied from 2.5 to $80 \%$. No effect was seen on the vertical eye velocity, whereas delayed, horizontal eye movements were strongly affected. Increasing the static contrast increased the initial horizontal eye velocity. $c$, Change in horizontal (top plot) and vertical (bottom plot) positions of ocular following responses, to either single grating (open symbols) or plaid pattern (closed symbols) motion, as a function of stimulus contrast. Changes in position are computed over the $95-115$ or the $95-135 \mathrm{msec}$ time window for grating or plaid motion conditions, respectively.

1988) as well as local edges and features motion (Löffler and Orbach, 1999).

The IOC and Fourier-non-Fourier models make different predictions for tracking initiation. First, the model proposed by Wilson et al. (1992) postulates different delays for Fourier and non-Fourier motions. Indeed, we show that grating- and patternrelated tracking components have different latencies, which is not predicted by the IOC model. Second, contrary to the IOC rule, the Fourier-non-Fourier model predicts that perceived direction has a time course that evolves from a linear combination of $1 \mathrm{D}$ motion signals toward the true object motion. Thus, at least over a limited period of time, there should be a persistent residual bias toward the Fourier motion direction (Yo and Wilson, 1992). With unikinetic plaids, we show that tracking shifts gradually from grating to pattern motion directions. Over the 135-175 msec interval, tracking direction is halfway between grating and pattern motion directions and is therefore close to the direction of the vector average of Fourier and non-Fourier motions, which is consistent with psychophysical results on perceived direction of short-duration unikinetic plaids (Wilson and Kim, 1994). Similarly, with barber-pole stimuli, tracking directions at the end of the open-loop period were best predicted by the vector sumaverage of grating and terminator motions (Masson et al., 2000).
Over a longer time interval however, more complex computational rules and closing of the visuomotor feedback loop can be involved so that, after several hundreds of milliseconds, tracking is perfectly aligned with object motion.

\section{Motion coherency, texture boundary processing, and parallel processing}

A key feature of moving plaids is that motion coherency breaks down when components differ sufficiently along one dimension, such as contrast, spatial frequency, binocular disparity, or color. Adelson and Movshon (1982) proposed that motion coherence is computed using the IOC rule within a single channel selective for these features. The two motion pathways model also predicts that the two component gratings must have similar spatial frequencies, otherwise the first stage filter for non-Fourier computation will respond to at most one of the components, but never both, and therefore no texture boundaries will be extracted, and nonFourier motion will never be computed (Kim and Wilson, 1993). Numerous psychophysical studies have demonstrated that gratings are detected almost completely independently if their spatial frequencies differ by $>1.5$ octaves (Blakemore and Campbell, 1969), and the channel model suggests a limit of coherence around this value (Smith, 1992). We found significant late com- 

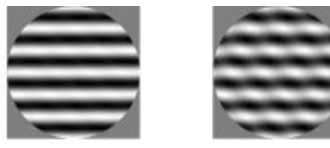

grating: early component

- plaid: late component

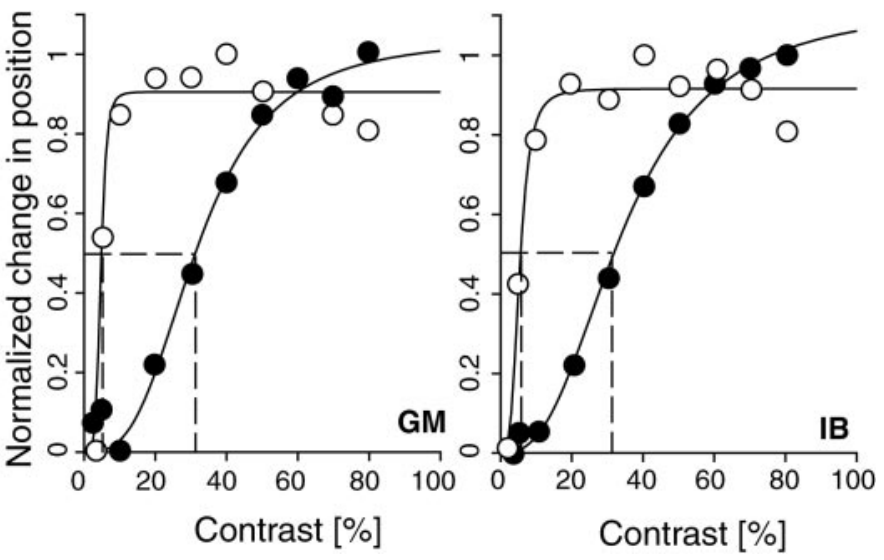

Figure 14. Contrast response functions. For two subjects, the normalized changes in position are plotted against contrast. Continuous lines are best-fit Naka-Rushton functions. Vertical dotted lines indicate the halfsaturation values of the contrast response functions. Open symbols plot the amplitude of early vertical responses, as a function of moving grating contrast. Closed symbols plot the amplitude of late, horizontal responses, as a function of static grating contrast.

ponent responses with large differences in spatial frequencies, up to three octaves, and largest late ocular following occurred when spatial frequencies differed by $\sim 1.5$ octaves. These results diverge significantly from psychophysical studies showing best coherency at a 1:1 ratio between spatial frequencies (Adelson and Movshon, 1982; Kim and Wilson, 1993). Such discrepancy could be explained if motion coherency was computed after a pooling of motion signals across a broad range of spatial frequencies (Smith, 1992). Alternatively, because we used a low spatial frequency moving grating $(0.27 \mathrm{cpd})$ and because low spatial frequency channels are more broadly tuned (Blakemore and Campbell, 1969), a larger bandwidth for motion coherency could be expected.

Relative contrast also determines motion coherency. Consistent with previous psychophysical studies, we found that maximal plaid-driven ocular following occurred for small contrast differences between static and moving gratings. Moreover, a rather high contrast of static grating was necessary to drive the late ocular component. Contrast response functions of early and late components were very different, as psychophysically observed for single grating and plaid motion detection, respectively (Adelson and Movshon, 1982).

When either relative spatial frequency or contrast is manipulated, late but not early ocular following is affected, suggesting that grating- and pattern-related motion inputs to the tracking system are processed independently. This result is consistent with the fact that changing either grating or line-ending motion independently affects the early and late phases of ocular following to barber-pole stimuli (Masson et al., 2000). These results are a strong argument for parallel processing of Fourier and nonFourier motion signals (Chubb and Sperling, 1988; Wilson et al., 1992).

\section{Neural mediation}

There is considerable evidence that, in monkeys, ocular following responses are mediated by cortical motion processing (Kawano,
1999). Single-unit recordings in monkey areas MT and MST have revealed directionally selective neurons that are activated by moving random dots at latencies that precede ocular following by $\sim 10$ msec (Kawano et al., 1994, 1997). Many of these MT and MST cells are sensitive to high speed motion (Kawano et al., 1994) and have a broad spatial selectivity and a high temporal resolution (Movshon and Newsome, 1996). These properties fit very well many of the characteristics of early ocular following in both monkeys (Miles et al., 1986) and humans (Gellman et al., 1990). Herein, we find that early ocular following exhibits a steep dependency on stimulus contrast with a very high sensitivity $\left(C_{50}\right.$ $\sim 5 \%$ ) but a very limited dynamical range. Very similar contrast dependencies were found for monkey MT neurons (Sclar et al., 1990), and in particular those receiving a direct projection from area V1 (Movshon and Newsome, 1996). Such contrast response functions are very similar to those of magnocellular geniculate neurons (Derrington and Lennie, 1984; Sclar et al., 1990), as expected from the predominantly magnocellular origin of the visual inputs to MT (Maunsell et al., 1990; Yabuta et al., 2001). Thus, fast inputs to areas MT and MST mediate the earliest ocular following.

What is the neural mediation of the late ocular following? We show that its latency is delayed by $\sim 20 \mathrm{msec}$, that it is specifically driven by pattern-related motion, and it has a broader dynamical range for contrast sensitivity. The latter properties might give a hint as to its neural substrate. With a $C_{50}$ of $\sim 30 \%$ and a very broad dynamical range, the contrast response functions plotted in Figure 14 are indeed very similar to those reported for parvocellular geniculate neurons and some complex cells in area V1 (Sclar et al., 1990). Moreover, they are also similar to the vast majority of direction-selective cells in monkey area V2 (Levitt et al., 1994). Wilson et al. (1992) have postulated that Fourier and non-Fourier pathways correspond to direct and indirect routes between areas V1 and MT. In fact, MT neurons respond to both plaids (Movshon et al., 1985; Rodman and Albright, 1989; Dobkins et al., 1998) and pure second-order motion (Albright, 1992; O'Keefe and Movshon, 1998). Interestingly, some MT neurons have similar orientation and direction selectivity for both first- and second-order motion, but contrast response functions are much steeper for the former (O'Keefe and Movshon, 1998). MT receives input both from areas V1 and V2, the latter being a crucial stage along the indirect route (Van Essen et al., 1992). In cat area 18 , responses to non-Fourier motions are delayed relative to Fourier motions (Mareschal and Baker, 1998). Similar evidence are lacking in primate, but it is known that the feedforward sweep of visually triggered neural activity reaches V2 20 msec later than MT (Lamme and Roelfsema, 2000). Finally, V2 lesions specifically impair the discrimination of non-Fourier boundary orientations (Merigan et al., 1993). These results suggest that the time course of ocular following might reflect the successive input of direct and indirect routes to MT. Our results open the door to future experiments on the relative contribution of these parallel motion pathways to tracking behavior.

\section{REFERENCES}

Adelson EH, Movshon JA (1982) Phenomenal coherence of moving visual pattern. Nature 300:523-525.

Albrecht DG, Hamilton DB (1982) Striate cortex of monkey and cat: Contrast response function. J Neurophysiol 48:217-237.

Albright TD (1992) Form-cue invariant motion processing in primate visual cortex. Science 255:1141-1143.

Blakemore C, Campbell FW (1969) On the existence of neurones in the human visual cortex selectively sensitive to the orientation and size of retinal images. J Physiol (Lond) 203:237-260. 
Boulton JB, Baker CL (1991) Motion detection is dependent on spatial frequency not size. Vision Res 31:77-87.

Busettini C, Miles FA, Schwartz U (1991) Ocular responses to translation and their dependence on viewing distance. II. Motion of the scene. J Neurophysiol 66:865-878.

Carl JR, Gellman RS (1987) Human smooth pursuit: stimulusdependent responses. J Neurophysiol 57:1446-1463.

Chubb C, Sperling G (1988) Drift-balanced random stimuli : a general basis for studying non-Fourier motion perception. J Opt Soc Am A 5:1986-2007.

Collewijn H, van der Mark F, Jansen TC (1985) Precise recordings of human eye movements. Vision Res 15:447-450.

Derrington AM, Lennie P (1984) Spatial and temporal contrast sensitivities of neurones in lateral geniculate nucleus of macaque. J Physiol (Lond) 357:219-240.

Dobkins KR, Stoner GR, Albright TD (1998) Perceptual, oculomotor and neural responses to moving color plaids. Perception 27:681-709.

Fennema CL, Thompson WB (1979) Velocity determination in scenes containing several moving images. Comp Graph Image Proc 9:301-315.

Ferrera VP (2000) Task-dependent modulation of the sensorimotor transformation for smooth pursuit eye movements. J Neurophysiol 84:2725-2738.

Ferrera VP, Wilson HR (1990) Perceived direction of moving twodimensional patterns. Vision Res 30:272-287.

Gellman RS, Carl JR, Miles FA (1990) Short-latency ocular following responses in man. Vis Neurosci 5:107-122.

Goréa A, Lorenceau J (1991) Directional performances with moving plaids: component- related and plaid-related processing modes coexist. Spat Vis 5:231-252.

Hays AV, Richmond BJ, Optican LA (1982) A UNIX-based multiple process system for real-time data acquisition and control. WESCON Conf Proc 2:1-10.

Heeger DJ (1987) A model for the extraction of optic image. J Opt Soc Am A 4:1455-1471.

Kawano K (1999) Ocular tracking: behavior and neurophysiology. Curr Opin Neurobiol 9:467-473.

Kawano K, Miles FA (1986) Short-latency ocular following responses of monkey. II. Dependence on a prior saccadic eye movement. J Neurophysiol 56:1355-1380.

Kawano K, Shidara M, Watanabe Y, Yamane S (1994) Neural activity in cortical area MST of alert monkey during ocular following responses. J Neurophysiol 71:2305-2324.

Kawano K, Inoue Y, Takemura A, Kitama T, Miles FA (1997) A cortically mediated visual stabilization mechanism with ultra-short latency. In: Parietal lobe contribution to orientation in 3D space (Thier $\mathrm{P}$, Karnath H-O, eds), pp 185-199. Heidelberg: Springer.

Kim J, Wilson HR (1993) Dependence of plaid motion coherence on component grating directions. Vision Res 33:2479-2489.

Lamme VA, Roelfsema PR (2000) The distinct modes of vision offered by feedforward and recurrent processing. Trends Neurosci 23:571-579.

Landy MS, Cohen Y, Sperling G (1984) HIPS: image processing under UNIX. Software and applications. Behav Res Methods Instrum Computers 16:199-216.

Levitt JB, Kiper DC, Movshon JA (1994) Receptive fields and functional architecture of macaque V2. J Neurophysiol 71:2517-2542.

Lisberger SG, Ferrera VP (1997) Vector averaging for smooth pursuit eye movements initiated by two moving targets. J Neurosci 17:7490-7502.

Löffler G, Orbach HS (1999) Computing feature motion without feature detectors: a model for terminator motion without end-stopped cells. Vision Res 39:859-871.

Lorenceau J, Shiffrar M, Wells N, Castet E (1993) Different motion sensitive units are involved in recovering the direction of moving lines. Vision Res 33:1207-1217.
Mareschal I, Baker CL (1998) Temporal and spatial response to secondorder stimuli in cat area 18. J Neurophysiol 80:2811-2823.

Masson GS, Rybarczyk Y, Castet E, Mestre DR (2000) Temporal dynamics of motion integration for the initiation of tracking eye movements at ultra-short latencies. Vis Neurosci 17:753-767.

Masson GS, Busettini C, Yang D, Miles FA (2001) Short-latency ocular following in humans: sensitivity to binocular disparity. Vision Res 41:3371-3387.

Maunsell JHR, Nealey TA, DePriest DD (1990) Magnocellular and parvocellular contributions to responses in the middle temporal visual area (MT) in the macaque. J Neurosci 10:3223-3334.

Merigan WH, Nealey TA, Maunsell JH (1993) Visual effects of lesions of cortical area V2 in macaques. J Neurosci 13:3180-3191.

Mestre DR, Masson GS (1997) Ocular responses to motion parallax stimuli : the role of perceptual and attentional factors. Vision Res 37:1627-1641

Miles FA (1998) The neural processing of 3-D visual information: Evidence from eye movements. Eur J Neurosci 10:811-822.

Miles FA, Kawano K, Optican LM (1986) Short-latency ocular following responses of monkey. I. Dependence on temporo-spatial properties of the visual input. J Neurophysiol 56:1321-1354.

Movshon JA, Newsome WT (1996) Visual response properties of striate cortical neurons projecting to area MT in macaque monkeys. J Neurosci 16:7733-7741.

Movshon JA, Adelson EH, Gizzi MS, Newsome WT (1985) The analysis of visual moving patterns. In: Pattern recognition mechanism (Chagas C, Gattass R, Gross C, eds), pp 117-151. New York: Springer.

Naka KI, Rushton WA (1966) S-potentials from color units in the retina of the fish. J Physiol (Lond) 185:584-599.

O'Keefe LP, Movshon JA (1998) Processing of first- and second-order motion signals by neurons in area MT of the macaque monkey. Vis Neurosci 15:305-317.

Pack CC, Born RT (2001) Temporal dynamics of a neural solution to the aperture problem in visual area MT of macaque brain. Nature 409:1040-1042.

Robinson DA (1963) A method of measuring eye movements using a scleral search coil in a magnetic field. IEEE Trans Biomed Eng 10:137-145.

Rodman HR, Albright TD (1989) Single-unit analysis of pattern-motion selective properties in the middle temporal visual area (MT). Exp Brain Res 75:53-64.

Sclar G, Maunsell JH, Lennie P (1990) Coding of image contrast in central visual pathways of the macaque monkey. Vision Res 30:1-10.

Smith AT (1992) Coherence of plaids comprising components of disparate spatial frequencies. Vision Res 22:393-397.

Smith AT, Harris LR (1991) Use of plaid patterns to distinguish the corticofugal and direct retinal inputs to the brainstem optokinetic nystagmus generator. Exp Brain Res 86:324-332.

Van Essen DC, Anderson CH, Felleman DJ (1992) Information processing in the primate visual system: an integrated systems perspective. Science 67:961-980.

Wilson HR (1999) Non-Fourier cortical processes in texture, form and motion perception. In: Cerebral cortex. Vol 13, Models of cortical circuits (Ulinsky PS, ed), pp 445-477. New York: Kluwer.

Wilson HR, Kim J (1994) A model for motion coherence and transparency. Vis Neurosci 11:1205-1220.

Wilson HR, Ferrera VP, Yo C (1992) A psychophysically motivated model for two-dimensional motion perception. Vis Neurosci 9:79-98.

Yabuta NH, Sawatari A, Callaway EM (2001) Two functional channels from primary visual cortex to dorsal visual cortical areas. Science 292:297-300.

Yo C, Wilson HR (1992) Perceived direction of moving twodimensional patterns depends on duration, contrast and eccentricity. Vision Res 32:135-147. 